\title{
Free Charge Photogeneration in a Single Component High Photovoltaic Efficiency Organic Semiconductor
}

Justin Hodgkiss ( $\square$ justin.hodgkiss@vuw.ac.nz )

Victoria University of Wellington

Michael Price

Victoria University of Wellington

Paul Hume

Victoria University of Wellington

Aleksandra llina

Victoria University of Wellington

Isabella Wagner

Victoria University of Wellington https://orcid.org/0000-0001-5009-2407

Ronnie Tamming

Victoria University of Wellington

Karen Thorn

Victoria University of Wellington

Wanting Jiao

Victoria University of Wellington

Alison Campbell

The University of Sydney

Patrick Conaghan

The University of Sydney

Girish Lakhwani

The University of Sydney

Nathaniel Davis

Victoria University of Wellington

Yifan Wang

Qingdao University

Peiyao Xue

Peking University

Heng Lu

Peking University

Kai Chen 
Victoria University of Wellington

\section{Xiaowei Zhan}

Peking University

\section{Physical Sciences - Article}

Keywords: photogeneration, solar energy

Posted Date: April 8th, 2021

DOI: https://doi.org/10.21203/rs.3.rs-376343/v1

License: (c) (i) This work is licensed under a Creative Commons Attribution 4.0 International License. Read Full License

Version of Record: A version of this preprint was published at Nature Communications on May 20th, 2022. See the published version at https://doi.org/10.1038/s41467-022-30127-8. 


\section{Free Charge Photogeneration in a Single Component High 2 Photovoltaic Efficiency Organic Semiconductor}

3 Michael B. Price ${ }^{\mathrm{a}, \mathrm{b}, 1, *}$, Paul A. Hume ${ }^{\mathrm{a}, \mathrm{b}, 1, *}$, Aleksandra Ilina $^{\mathrm{a}, \mathrm{b}}$, Isabella Wagner ${ }^{\mathrm{a}, \mathrm{b}}$, Ronnie R.

4 Tamming $^{\mathrm{a}, \mathrm{b}}$, Karen E. Thorn ${ }^{\mathrm{a}, \mathrm{b}}$, Wanting Jiao ${ }^{\mathrm{c}}$, Alison Campbell ${ }^{\mathrm{d}}$, Patrick J. Conaghan ${ }^{\mathrm{d}}$, Girish 5 Lakhwanid $^{\mathrm{d}}$, Nathaniel J.L.K. Davis ${ }^{\mathrm{a}, \mathrm{b}}$, Yifan Wang, ${ }^{\mathrm{e}, \mathrm{f}}$, Peiyao Xue ${ }^{\mathrm{e}}$, Heng Lu $^{\mathrm{e}}$, Kai Chen ${ }^{\mathrm{a}, \mathrm{b}}$, 6 Xiaowei Zhan ${ }^{\mathrm{e}}$, Justin M. Hodgkiss ${ }^{\mathrm{a}, \mathrm{b}, *}$

7

8

9

${ }^{a}$ School of Chemical and Physical Sciences, Victoria University of Wellington, Wellington, New Zealand

${ }^{\mathrm{b}}$ MacDiarmid Institute for Advanced Materials and Nanotechnology, New Zealand.

${ }^{\mathrm{c}}$ Ferrier Research Institute, Victoria University of Wellington, Wellington, New Zealand

${ }^{\mathrm{d} A R C}$ Centre of Excellence in Exciton Science, School of Chemistry, University of Sydney, NSW 2006, Australia

${ }^{\mathrm{e}}$ School of Materials Science and Engineering, Peking University, Beijing 100871, China.

${ }^{\mathrm{f}}$ College of Materials Science and Engineering, Qingdao University, Qingdao 266071, China

* Correspondence should be sent to J.M.H. (Justin.Hodgkiss@ vuw.ac.nz), P.A.H.

(Paul.Hume@ vuw.ac.nz), and M.B.P. (Michael.price@vuw.ac.nz)

${ }^{1}$ Authors contributed equally to this work

\section{ABSTRACT}

Organic photovoltaic cells promise cheap, flexible and scalable solar energy. ${ }^{1,2}$ Whereas light directly generates free charges in silicon photovoltaic cells, bound electron and hole pairs known as excitons are understood to be the primary excitations in organic semiconductors due to their low dielectric constants. ${ }^{3}$ These excitons must then be split apart at molecular heterojunctions in order to extract current. ${ }^{4}$ Recent record efficiency organic photovoltaics utilise the small molecule, Y6, ${ }^{5-8}$ as a key component in the photon-absorbing blend layer. This molecule and its analogues - unlike previous organic semiconductors - have both low bandgaps and high dielectric constants. ${ }^{9}$ Here we show that, in a neat film of Y6, these factors lead to intrinsic free charge generation without the need for a molecular heterojunction to split the exciton. We use a suite of intensity-dependent optical spectroscopy measurements to show that a significant (20-90\%) fraction of free charges exist in equilibrium with bound states at light intensity equivalent to 1 sun. Rapid bimolecular charge recombination constrains single 
component Y6 organic photovoltaic devices to low efficiencies, but this recombination is reduced by the introduction of small quantities of donor polymer. Quantum-chemical calculations reveal charge generation pathways through strong coupling between exciton and CT states, and an intermolecular polarisation pattern that drives exciton dissociation. Our results challenge the understanding of how current record efficiency organic photovoltaics operate, and point towards new future possibilities - offering a molecular picture of intrinsic charge generation as a platform to improve charge yields, and renewing the possibility of efficient single-component organic photovoltaic devices.

\section{INTRODUCTION}

When light is absorbed by inorganic semiconductors, like silicon or gallium arsenide, a combination of free charges and excitons (bound electrons and holes) are created, and their ratio depends upon the material's dielectric constant. For organic semiconductors, the paradigm holds that only excitons are intrinsically photogenerated because the low dielectric constant ${ }^{10,11}$ of these materials $(\varepsilon \sim 3-4)$ means the electron and hole Coulombic interaction is not efficiently screened, leading to Frenkel excitons with high binding energies $\left(E_{\mathrm{B}}\right)$. For the last thirty years, ${ }^{4,12}$ this constraint has guided the development of organic photovoltaics (OPVs) which promise a step-change in flexible, lightweight, non-toxic solution-processed solar energy production, but are yet to be widely commercialised. Splitting bound excitons into free charges has required sharp molecular heterojunctions between donor and acceptor materials (though some have recently shown field-assisted exciton dissociation, or CT-state/polaron-pair formation $\left.{ }^{13-16}\right)$. Rather than purely optimising charge harvesting, OPV devices have been optimised for exciton splitting at interfaces, and demand complex interpenetrating networks of donor and acceptor materials. The molecular heterojunction approach limits device efficiencies, ${ }^{17}$ introduces inherent voltage losses and instabilities from interfaces, ${ }^{18}$ and complicates research progress. ${ }^{19}$

Recently, small molecule non-fullerene fused ring electron acceptors (FREAs) ${ }^{3,6,20-24}$ have driven a rapid uptick in the power conversion efficiency (PCE) of OPVs. Alongside this advance has arisen unexpected observations, namely: 1) Barrierless free charge generation in PM6:Y6 blends ${ }^{25}$ (full chemical names and structures shown in Figure S1) 2) That charge generation efficiency in blends of PM6:Y6 increases with increasing incident light intensity. ${ }^{26}$ 3) In $\mathrm{Y}^{16,27}$ and IDIC, ${ }^{28}$ excitons are delocalised, or form an 'intra-moiety' intermediate state 
with likely charge-transfer (CT) like character. These observations have all been explained by invoking CT-states, however, with additional data, we show here that a more profound explanation is required.

FREAs have recently been measured to have very high refractive indices, ${ }^{29,30}$ and hence high complex dielectric constants. Of the FREAs, Y6 and its derivatives are present in both the binary and ternary OPVs that hold the highest PCEs. ${ }^{5-8}$

In inorganic semiconductors, the number of bound excitons versus free charges can be described by the Saha-Langmuir relation.

$$
\frac{x^{2}}{1-x}=\frac{1}{n}\left(\frac{2 \pi m^{*} k_{B} T}{h^{2}}\right)^{1.5} e^{-\frac{E_{B}}{k_{B} T}}
$$

Where the free charge fraction, $x$, is dependent upon the temperature, $T$, excitation density, $n$, effective mass, $m^{*}$ and the exciton binding energy, $E_{B}$ (which is related to the inverse of the dielectric constant ${ }^{31}$ ). From comparison of the highest occupied molecular orbital (HOMO)lowest unoccupied molecular orbital (LUMO) gap to the optical gap measured by Karuthedath et al., ${ }^{18}$ the exciton binding energy of $\mathrm{Y} 6$ is $E_{B}=100-250 \mathrm{meV}$ - much lower than for example, P3HT $\left(E_{B} \sim 700 \mathrm{meV}\right){ }^{32}$ Based on a midpoint, $175 \mathrm{meV}$ binding energy, estimated for Y6 above, and assuming an effective mass between ${ }^{33,34} m^{*}=1.7-0.2 m_{e}$, this simple calculation would predict a high fraction (20-80\%) of intrinsically generated free charges.

Here, we show that the high optical frequency dielectric constant of Y6 leads to free charge generation upon optical excitation within neat films. We use a combination of ultrafast transient absorption, photoluminescence up-conversion and time-resolved terahertz spectroscopy, and identify the spectral signatures and decay rates of singlet excitons and free charges (polarons). Intensity-dependent photoluminescence quantum efficiency (PLQE) measurements prove the existence of radiative bimolecular charge recombination, which not be the case if only CT states were formed. We present kinetic models that reproduce our experimental data, wherein a free charge fraction of between 0.2-0.9 exists in equilibrium with bound charges under steady-state illumination. Fast bimolecular charge recombination inhibits the efficiency of single-component devices, but this recombination can be slowed by doping our Y6 films with only a small amount of 'p-type' polymer electron donor. Using quantum-chemical calculations, we show that free charge generation is expected due to strong coupling between equienergetic exciton and CT states, and reveal that asymmetric polarisation of the different lattice positions results in a "donor/acceptor" system derived purely from intermolecular interactions. The 
observation of intrinsic charge generation in a single organic material necessitates an expanded understanding of the photovoltaic process in organic materials. The implications for device design are far-reaching, showing that a new approach to reaching maximum efficiency may be to maximise intrinsic charge generation yield, whilst reducing rapid bimolecular charge recombination, rather than focus on exciton splitting in a donor-acceptor blend. Indeed, we suggest that the highest efficiency OPV blends (such as PM6:Y6) have in fact been optimised for the extraction of intrinsically formed charges.

\section{MAIN}

\section{Radiative bimolecular recombination}

Figure 1a shows the excitation density-dependent external photoluminescence quantum efficiency (PLQE) of a neat film of Y6 (BTP-4F). As the intensity of pulsed excitation is increased, the PLQE remains constant at $0.9 \%$ from $10^{14}$ to $10^{15}$ excitations per cubic $\mathrm{cm}$, before rising to a peak of $1 \%$ at a density of $\sim 10^{16} \mathrm{~cm}^{-3}$. Figure $1 \mathrm{~b}$ illustrates the expected behaviour of excitation density-dependent PLQE for varying proportions of radiative monomolecular to bimolecular processes. The rise in efficiency observed in Fig 1a is not possible for a system undergoing monomolecular (geminate) radiative recombination, even from intra-moiety CT states, and proves that a significant fraction of photoexcited species are following a pathway that involves bimolecular recombination prior to, or concurrently with, a radiative transition. As exciton-exciton annihilation can only produce a non-radiative bimolecular contribution, we conclude that charges must be present in the neat film, and that these charges are formed 'intrinsically', rather than through exciton annihilation processes. Under steady-state illumination, this free charge (polaron) population will exist in equilibrium with a population of bound excitons, and potential 'CT-like' states, as illustrated by Figure 1c. 

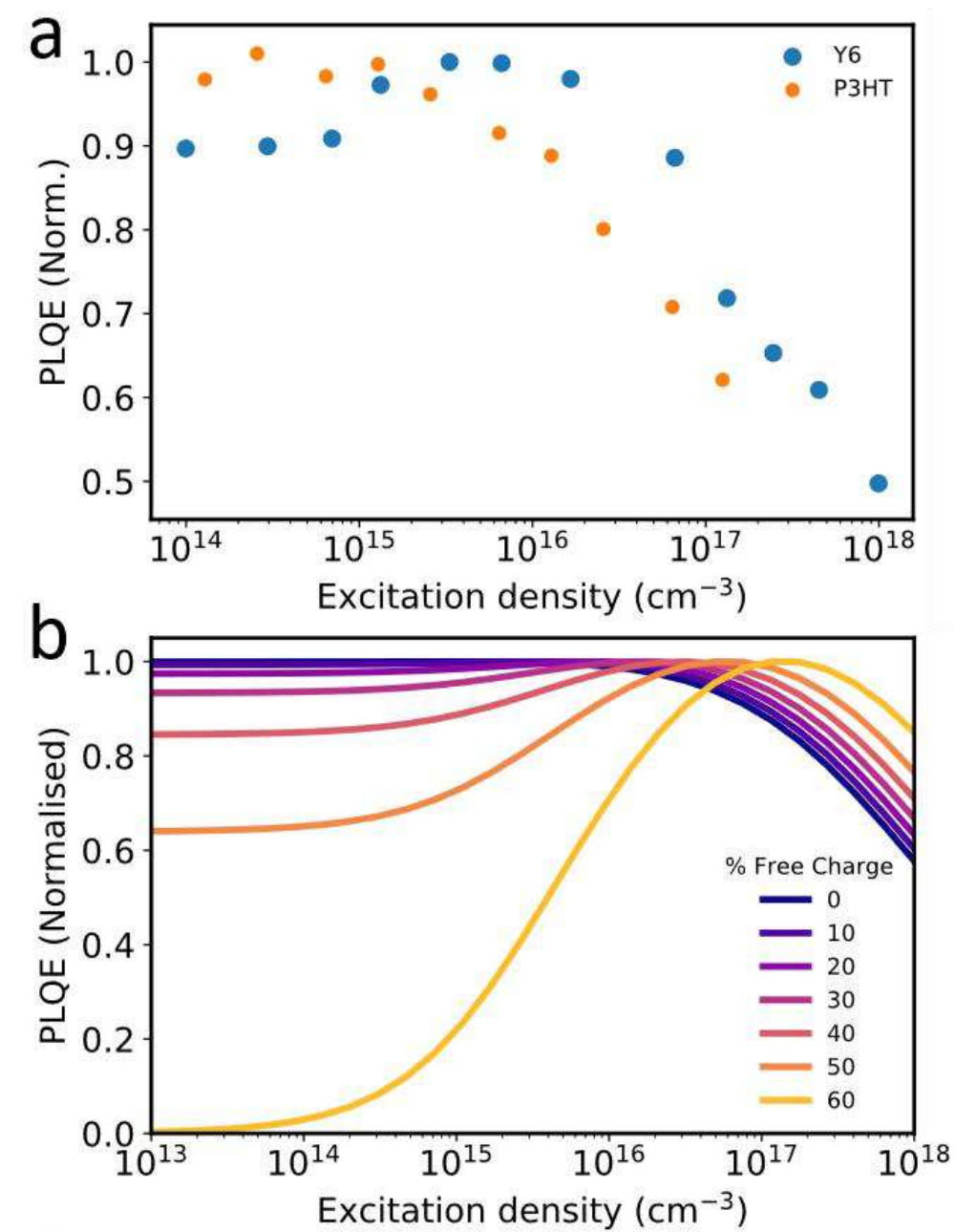

C

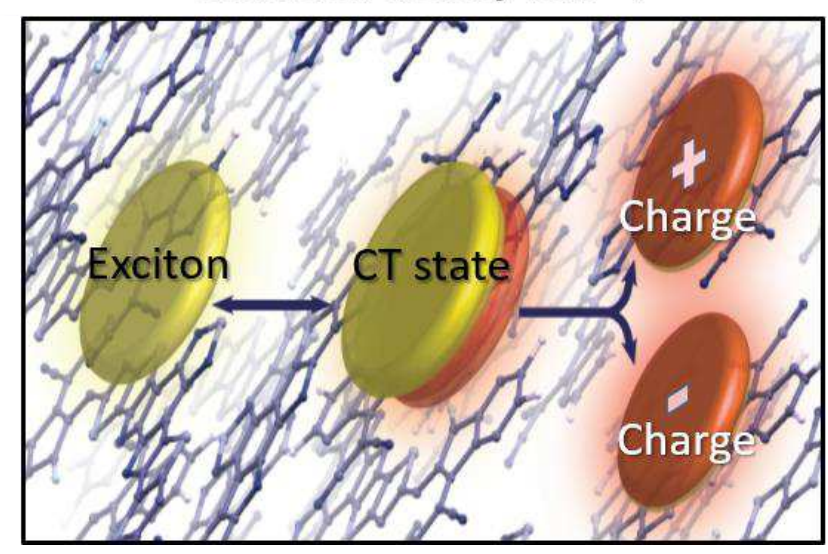

Figure 1. Intensity-dependent photoluminescence quantum efficiency of Y6.

a) Experimentally determined PLQE of a neat film of Y6 on glass substrate in an integrating sphere (normalised). The sample was excited by $600 \mathrm{ps}$ pulses of $532 \mathrm{~nm}$ light. The PLQE peaks at $1 \%$ at $\sim 10^{16}$ excitations $/ \mathrm{cm}^{3}$. Figure $\mathrm{S} 1$ gives an indication of the error associated with the data. Intensity dependent PLQE of a film of P3HT is shown as orange circles, as comparison, to illustrate the expected response. b) Simulation of intensity-dependent PLQE values showing effect of increasing (orange) or decreasing (blue) radiative bimolecular recombination (from prompt free charge fraction, calculated using Model 2 detailed in the SI). c) Graphic representation of exciton-CT state-free charge equilibrium in Y6. 
131 Exciton - charge dynamics and equilibrium

132 To quantify the relative proportions of charges versus excitons, we perform ultrafast 133 spectroscopic measurements on films of neat Y6. We identify spectral signatures of the singlet 134 exciton, and free charge/CT-state species and their kinetics to estimate first and second order 135 kinetic rates/lifetimes. Figure 2a shows the area-normalised transient absorption (TA) spectrum 136 of a neat Y6 film pumped with a $150 \mathrm{fs}$, $700 \mathrm{~nm}$ laser pulse at a moderate excitation density of $13710^{15}$ excitations $/ \mathrm{cm}^{3}$. The spectrum evolves from one initial photoexcited species, a sharp 138 negative peak at $915 \mathrm{~nm}$, to another consisting of both positive and negative features, apparent 139 after $\sim 500$ ps. Singular value decomposition, multivariate curve resolution analysis and 140 application of a genetic algorithm $(\mathrm{GA})^{35}$ consistently resolve two major species present, 141 shown as the blue and orange spectra in Figure 2b. We confirm that the orange species is that 142 of the polaron - referred to as free charge (FC) for consistency in the rest of the manuscript 143 by comparing the spectra of TA measurements of Y6 blended with either hole or electron 144 accepting species - PTB7-Th and poly-TPD respectively. We note that the charge is also likely 145 to have a very similar spectrum to an intraspecies charge- $\operatorname{transfer}^{36}$ state/excimer/weakly146 bound exciton delocalised over 2 or more molecules in the Y6 crystal, and therefore designate 147 the spectra here as representing the free charge plus CT state populations. We stress that we 148 cannot explain all of our data unless there is a significant fraction of free charges, as detailed 149 below. 

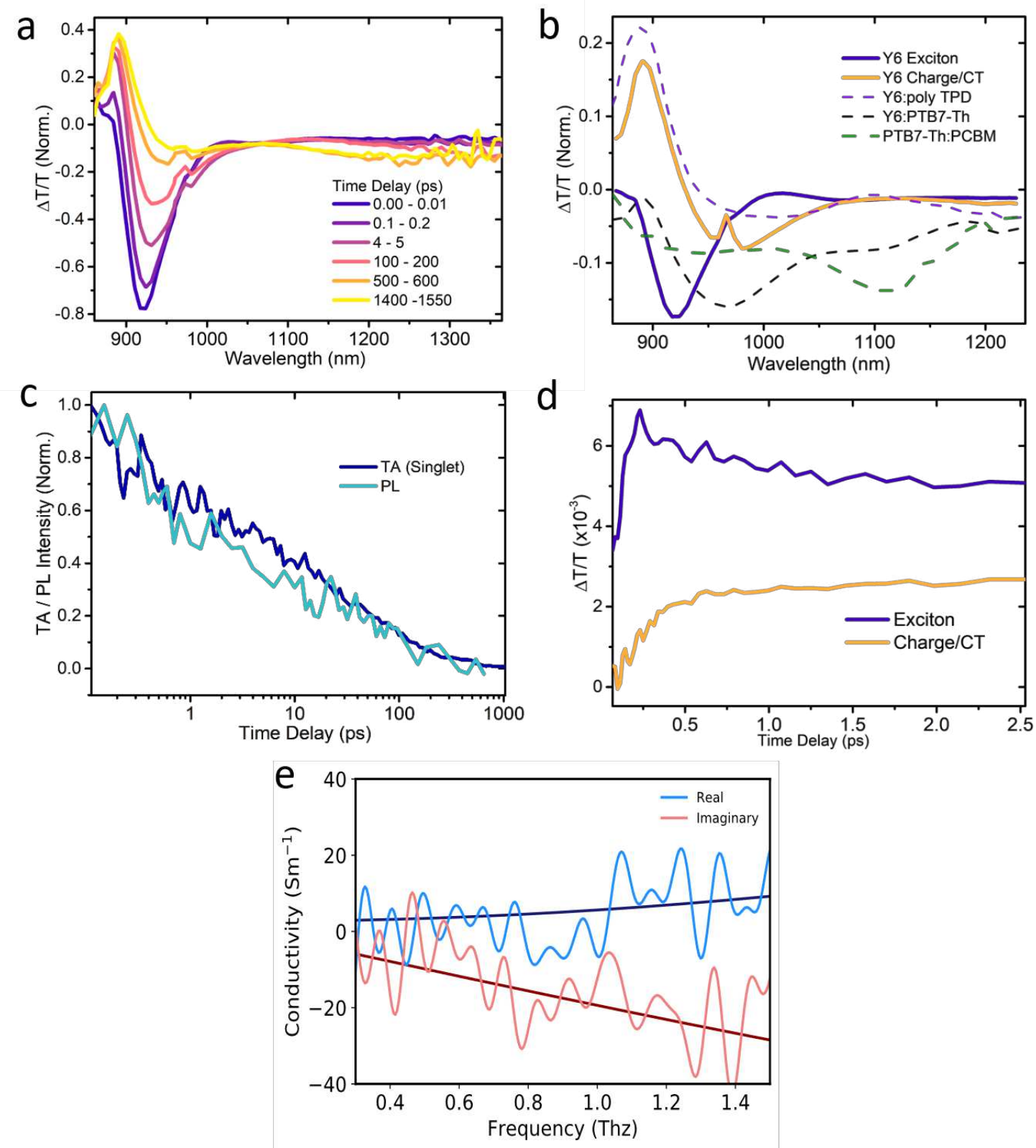

151 Figure 2. Ultrafast transient absorption spectra and kinetics of singlets and free charges in neat $\mathrm{Y6}$, and optical pump terahertz probe conductivity.

153 a) Transient absorption spectra, normalised to the absolute area integral, of a neat film of Y6 at different time delays. $700 \mathrm{~nm}, 200 \mathrm{fs}$ pump pulse, excitation density $=10^{15}$ excitations $/ \mathrm{cm}^{3}$. The spectrum evolves from the exciton, blue line, with a negative peak at $915 \mathrm{~nm}$, to the redshifted charge/CT state spectrum, orange line. b) Confirmation of charge and exciton transient absorption spectra by comparison of neat species (extracted using genetic algorithm) with species resolved from blends of Y6 with hole and electron accepting materials (PTB7-Th, polyTPD). The green dashed line shows the hole spectra of PTB7-Th blended with PCBM. c) Singlet component from GA of transient absorption (dark blue line), compared with upconverted PL signal (cyan), at an excitation density of $8 \times 10^{14} \mathrm{~cm}^{-3}$. d) Initial kinetics of the exciton (blue) versus charge/CT (orange) TA kinetics in the first 2.5 ps after photoexcitation. The charge species shows a rise concomitant with the fast initial decay of the singlet species. e) Excited-state terahertz complex conductivity spectrum, excited with $150 \mathrm{fs}, 800 \mathrm{~nm}$ light, 
giving approximate excitation density $5 \times 10^{15}$ excitations $/ \mathrm{cm}^{3}$, with a $\mathrm{THz}$ probe delay of $\sim 3$ ps. The positive real (light blue) line is indicative that there are charge-like species present in addition to purely excitonic photoexcitations. The experimental data is fit to a modified DrudeSmith model (darker blue and darker red curves).

We confirm that the blue species is that of the singlet exciton in 2 ways: 1) through TA measurements of a 'solid-solution' of Y6 diluted in polystyrene (Figure S2), and 2) by comparing the TA spectra of the 'blue' species to the transient kinetics of upconverted PL signal from the same film at similar excitation density. As shown in Figure $2 c$, the singlet TA matches the transient PL signal, confirming our assignment of the 'blue' species as the emissive singlet. The singlet, and free charge/CT species show different kinetic decays to each other across multiple different excitation density regimes (see Figure $3 b$ ).

The singlet and charge species, as shown in Figure $2 \mathrm{~d}$, show a prompt, fluence independent (Fig. S3), interconversion within the first 2.5 picoseconds, which also has no clear excitation-energy dependence (Fig. S4). These observations are consistent with observations from Wang et al., ${ }^{16}$ though our other measurements (Terahertz spectroscopy and fluence dependent PLQE) necessitate a model beyond pure CT generation. We quantify the initial prompt generation (within the first $2.5 \mathrm{ps}$ ) of charge/CT species in two ways - from the initial fast decay of the singlet, and from an estimation of the charge/CT visible TA bleach crosssection at $650 \mathrm{~nm}$. These methods both estimate that by $2.5 \mathrm{ps}$ after photoexcitation, $\sim 30 \%$ of initially excited singlets are rapidly converted to the charge/CT species. As this effect appears not to be due to excess energy (based on TA measurements pumped with different excess energies), the rapid formation is likely an effect of increased exciton delocalisation at early times. ${ }^{37,38}$ In line with Gillet et al., ${ }^{39}$ we also observe a small $3^{\text {rd }}$ spectral component in the TA which is consistent with a triplet signature (Fig. S5). As further evidence that there are significant free charges present, the triplet signal peak is significantly delayed compared to the charge/CT signal, which is consistent with a non-geminate triplet generation pathway rather than geminate triplet formation from inter-system crossing.

As another probe of free charge generation in Y6, we perform optical-pump-terahertz probe (OPTP) spectroscopy. ${ }^{40,41}$ Figure $2 \mathrm{e}$ shows the conductivity spectrum at 3 ps probe delay of a neat film of Y6, excited with an $800 \mathrm{~nm}$ pump beam, at an excitation density of $\sim 5 \times 10^{15}$ excitations $/ \mathrm{cm}^{3}$. The non-zero real part of the terahertz $(\mathrm{THz})$ spectrum (light blue line) is 
indicative of a fraction of free polarons, rather purely bound excitons. ${ }^{40,42,43}$ The conductivity spectrum is well described by a fit to a modified Drude-Smith model (DSM) - an established description for charge carriers in pi-conjugated materials. ${ }^{40,42,43}$

$$
\tilde{\sigma}(\omega)=\frac{\varepsilon_{0} \omega_{P}^{2} \tau}{1-i \omega \tau}\left[1+\frac{c_{1}}{1-i \omega \tau}\right]
$$

Where $\varepsilon_{0}$ is the vacuum permittivity, $\omega_{P}$ the plasma frequency, $\tau$ the scattering time, and $c_{1}$ is the 'localisation' parameter. A best fit to our data is obtained with a scattering time of $\tau=12 \mathrm{fs}$, a plasma frequency of $\omega_{P}=20 \mathrm{Thz}$, and $c_{1}=-0.99$. The localisation parameter indicates carriers that are well-localised, consistent with other reports in small molecules and conjugated polymers, and with our computational results presented below. The fitted plasma frequency, $\omega_{P}{ }^{2}=\frac{n q^{2}}{\varepsilon_{0} m_{e}}$, can be used to estimate an upper bound on the effective mass. Even assuming $100 \%$ charge yield, this gives a low effective mass of $m^{*}=0.16 m_{e}$, a value below those estimated in various non-fullerene molecules from DFT models. ${ }^{33}$ These results are again indicative of intrinsic charge generation, with rapid bimolecular recombination due to high local charge mobilities. ${ }^{40}$

We can fit our transient absorption kinetics and intensity-dependent PLQE data based on a basic kinetic model of free charges, charge-transfer states, and singlet excitons present in a neat film of Y6 upon illumination. Figure 3a graphically presents this model, along with rate estimates from application of the model to our experimental TA and PLQE data (details in SI and in Figure $3 \mathrm{~b}$ and $\mathrm{c}$ ). After the fast initial generation of CT/free charge states, the ratio of species present is governed by their interconversion rates: Singlet excitons can convert to CT states and vice versa, and CT states split easily into free charges, which can recombine bimolecularly back into CT states. 

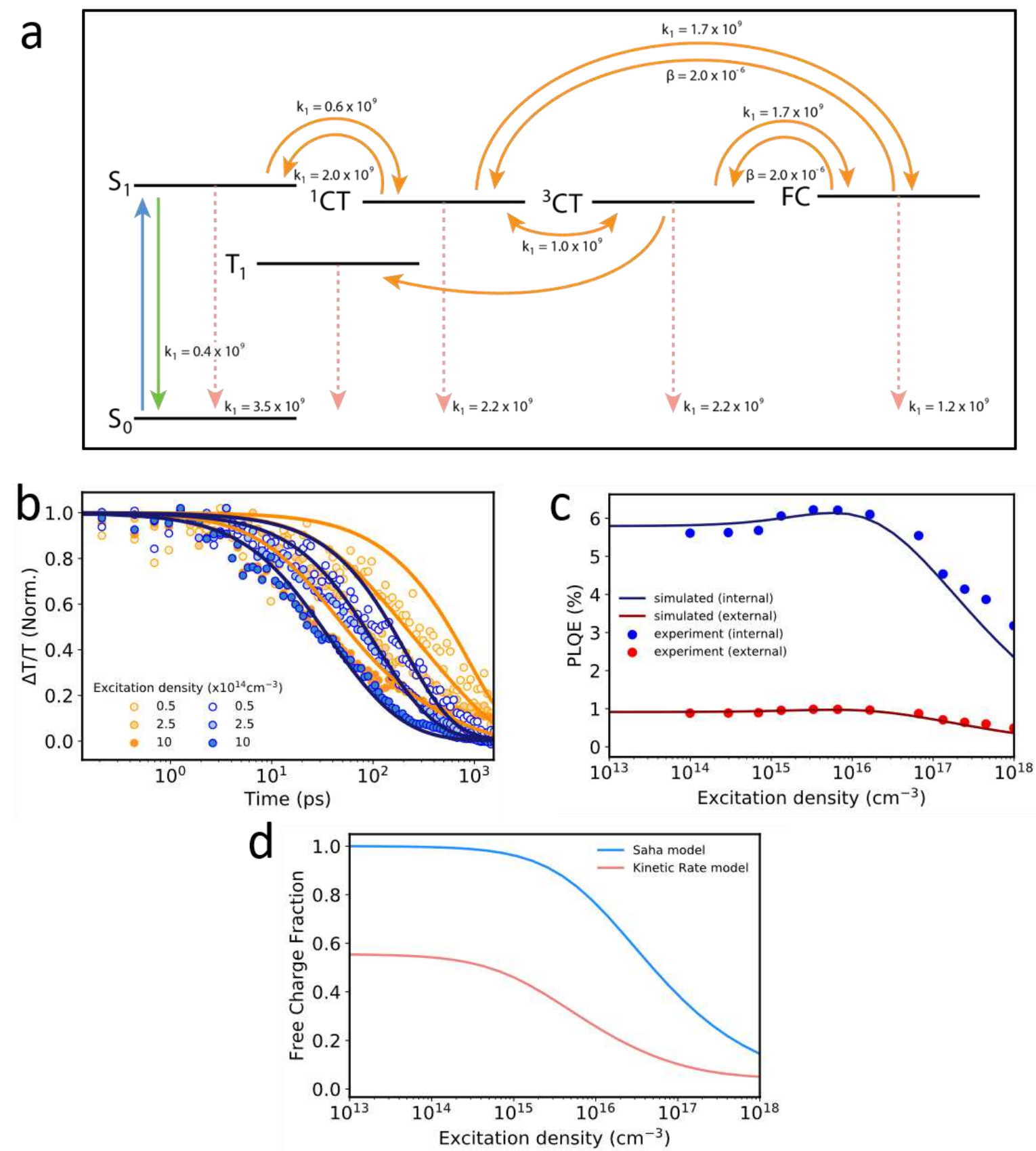

Figure 3. Kinetic model of charge generation in Y6 with transient absorption, PLQE and predicted steady-state free charge fractions. a) Jablonski diagram highlighting key excitedstate pathways, with associated rate constants. Monomolecular, $k_{l}$, values are given in units s ${ }^{-1}$, and bimolecular, $\beta$, values given in $\mathrm{cm}^{3} \mathrm{~s}^{-1}$. b) Normalised transient absorption kinetics of excitons (blue circles) and charges+CT states (orange circles) at different excitation densities, fitted with a global fit to the kinetic model described in the text and SI (solid blue and orange lines). c) Internal (blue) and external (red) PLQE values of Y6 as a function of excitation density, with corresponding simulated values from fits to the transient absorption and intensity dependent PLQE. Internal PLQE is calculated from external PLQE as per Richter et al. ${ }^{44} \mathrm{~d}$ ) Steady-state free charge fraction as a function of total excitation density calculated from the rate constants gathered from the kinetic model (red line), and from an estimate from the Saha equation (blue line). 
Figures $3 b$ and $3 c$ show the results of a global fit of this model to a multiple fluence series of transient absorption, and PLQE data. We calculate internal PLQE ${ }^{44}$ (taking photon recycling into account - see below) as, $\eta_{\text {int }}=\frac{d S_{\text {rad }}}{d N_{\text {total }}}$, where $d S_{\text {rad }}$ is the radiative rate of singlet decay, and $d N_{\text {total }}$ is the total decay rate of all species in the film. The radiative and nonradiative singlet decay rates are obtained from comparison of the PLQE values of Y6 in a solidstate solution of polystyrene, and the intersystem CT crossing rate is estimated from literature. ${ }^{45}$

This model simultaneously approximates the transient absorption data, and crucially, recreates the increase, or 'hump', in PLQE that is the signature of free charge generation. We thus view it as instructive for estimating free charge yields and transition rates in Y6. We note that we can also globally fit the data with an even simpler model, that does not explicitly include CT states, and which gives a higher $(\sim 75 \%)$ steady-state fraction of free charge population (Fig S8), and lower, likely more accurate estimate of bimolecular charge recombination rate though the rate is still high $\left(6 \times 10^{-8} \mathrm{~s}^{-1} \mathrm{~cm}^{3}\right)$. Error analysis of these models is shown in the supplementary information. Though there is significant interdependency between the fitted rates, and hence the individual error in each rate constant is high (and model dependent), we can use the models to put a lower and upper bound on steady-state free charge yield at a given excitation intensity. Setting the conservative criteria that there is a rise in PLQE with excitation intensity (of any magnitude), to match our experiments, the lower bound for steady-state free charge fraction, at an excitation density of $10^{16} \mathrm{~cm}^{-3}$ is $25 \%$ (our estimated solar excitation density is $5 \times 10^{16} \mathrm{~cm}^{-3}$ under the AM1.5 spectrum). The upper bound for both models is 80-90\%. A model that includes only singlet excitons and CT states cannot reproduce our PLQE data.

The red line in Figure 3d illustrates the fraction of free charges present at a steady state condition based on the model and rates described in Fig 3a, as a function of total excitation density. It mirrors the behaviour expected from using the Saha equation (blue line), with an effective mass of $1.7 \mathrm{~m}_{\mathrm{e}}$ and exciton binding energy, $E_{B}=175 \mathrm{meV}$. It shows that at low excitation densities, below $10^{15}$ excitations $/ \mathrm{cm}^{3}$, over $50 \%$ of excitations exist as free charges ( $60 \%$ of which arise from prompt generation within the delocalised state), and this portion drops as the density is increased.

\section{High photon reabsorption in thin films}

As illustrated in Figure 4c, it is important to take into account photon reabsorption in PLQE measurements of Y6 thin films. This is a further consequence of their extremely high 
refractive index. We use the same process as Richter et al. ${ }^{44}$ to approximate photon reabsorption, and show that the true PLQE of Y6 is much higher ( 6\%) than what would be ascertained from a direct reading of the external PLQE $(\sim 1 \%)$. This has significant importance for solar cell material optimisation efforts, as maximising PLQE is a useful approach for assessing photovoltaic efficiency potential, ${ }^{46}$ and ultimately, being able to harness radiative carrier recombination through photon recycling is key to approaching the Shockley-Quiesser limit. Although we estimate photon recycling is small for these measurements (Fig S8), The high refractive index means that it will become significant if internal PLQE can be increased.

\section{Fast bimolecular recombination in single component OPV devices}

The results from the transient absorption, PLQE, and terahertz spectroscopy data explain why, even though charges are intrinsically generated, single component, monolayer devices are not efficient. High bimolecular charge recombination rates mean that free charges recombine before arriving at electrodes in a device. With our measured charge recombination rates, and assuming (for the sake of illustration), pure Langevin recombination, at 1 sun intensity the charge diffusion length would be less than $10 \mathrm{~nm}$. We fabricated single component Y6 devices. The highest PCE based on a PEDOT:PSS/Y6/LiF/Al structure was $0.09 \%$. By replacing PEDOT:PSS with PCP-Na, whose HOMO better aligns with that of Y6, the highest PCE obtained was $0.63 \%$ (Fig S12). This value is low (though there is no built-in voltage in the active layer). This loss of photocurrent and photovoltage is due in significant part to high bimolecular recombination, which we show by performing intensity dependent short-circuit current $\left(\mathrm{J}_{\mathrm{SC}}\right)$ measurements. Previous reports have seen power law dependence of $\mathrm{J}_{\mathrm{SC}}$ on illumination intensity $(I)$ according to ${ }^{47,48}: \mathrm{J}_{\mathrm{SC}} \propto I^{\alpha}$. Deviations of the fitting parameter, $\alpha$, from unity signify bimolecular recombination or space-charge effects due to unbalanced charge mobilities. ${ }^{47-50}$ Electron and hole mobilities are relatively balanced in Y6 (being $1.8 \times 10^{-4}$ and $5.6 \times 10^{-4} \mathrm{~cm}^{2} \mathrm{~V}^{-1} \mathrm{~s}^{-1}$ respectively $\left.{ }^{27}\right)$, and at intensities below 0.01 suns, the exponent, $\alpha$, approaches 1 (Fig S13), indicating that space-charge losses could be limited. Figure 4a shows that for the region between 0.2 and 2 suns incident photo-intensity, the fitted exponent, $\alpha=0.84$, indicating the device suffers from significant bimolecular recombination at 1 sun.

To further emphasise the importance of high bimolecular recombination, rather than exciton splitting, as a limiting factor in device efficiency, we investigated the effect of doping our Y6 material with very small amounts of donor polymer, PTB7-Th. ${ }^{51}$ We performed transient absorption spectroscopy on these blends, and measured the photovoltaic device 
external quantum efficiencies of the corresponding devices. As shown in Figure $4 b$, when blended in a 1:50 ratio of PTB7-Th:Y6, the EQE is nearly twice as high as for the 1:100 parts blend. However, Figure $4 \mathrm{c}$ shows that this is not due to inefficient exciton splitting. The transient absorption kinetics show that for both blends the exciton species decay, and the charge species appear, with almost identical kinetics in the first $20 \mathrm{ps,} \mathrm{but} \mathrm{the} \mathrm{charges} \mathrm{recombine} \mathrm{faster}$ in the 1:100 blend, showing that the polymer donor's principal role is to decrease the rate of recombination, rather than split excitons for this system.
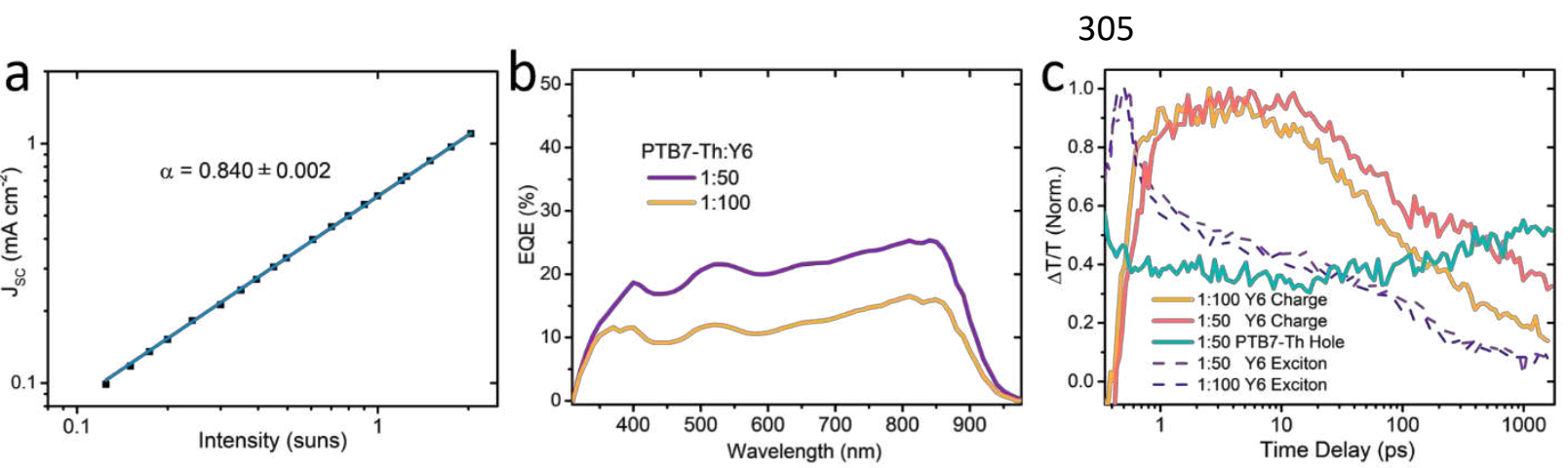

Figure 4. Single component short circuit current, and low donor active layer device EQEs, and corresponding exciton and charge kinetics. a) Intensity dependent $\mathrm{J}_{\mathrm{SC}}$ of a monolayer Y6 device. The power exponent fitted to the data between 0.2 and 2 suns is 0.7 , indicating a high number of bimolecular recombination events. b) External quantum efficiencies (EQEs) of devices with active layers consisting of small ratios of donor, PTB7-Th, to acceptor, Y6. For such low donor content, EQEs are unexpectedly high. c) Normalised transient absorption kinetics of exciton and charge species in blends of PTB7-Th:Y6 with ratios of 1:50 and 1:100. Pumped with $800 \mathrm{~nm}, 150$ fs pulses at excitation density $1 \times 10^{14}$ excitations $/ \mathrm{cm}^{3}$. The exciton kinetics of both films, dashed lines, are matching, as are the rise times of the charge kinetics (orange and red lines), but the 1:50 blend shows slower charge recombination after $20 \mathrm{ps}$. The green-blue line shows the hole kinetics in the 1:50 blend, showing a gradual increase in hole population as excitons and charges in Y6 diffuse to the PTB7-Th.

\section{Molecular picture of charge generation}

Having shown the macroscopic properties necessary for intrinsic charge generation, density functional theory calculations were used to gain an understanding of the structural features responsible for charge generation in Y6. The first requirement is significant electronic coupling between $\mathrm{S}_{1}$ and $\mathrm{CT}_{1}$. This parameter was calculated for the three molecular pairs in the Y6 crystal structure ${ }^{27}$ exhibiting $\pi-\pi$ stacking interactions using our previously described projective method, ${ }^{52,53}$ which enables us to simultaneously predict the energies of localised exciton/CT states, and the couplings between them (Figure 5 a). 
a

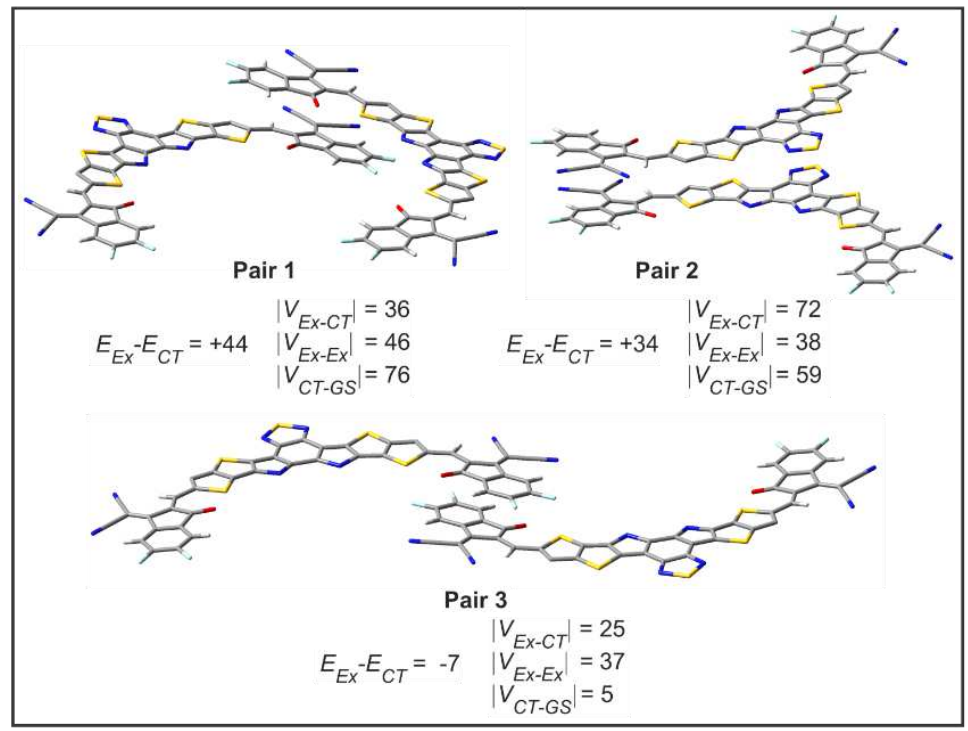

b

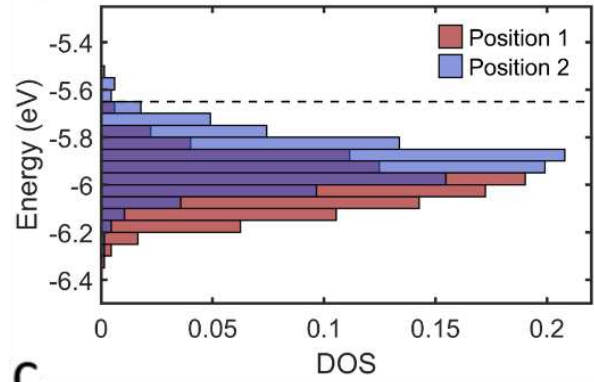

C

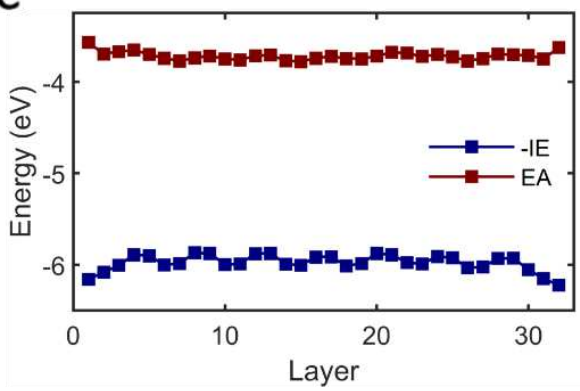

Figure 5. Electronic couplings and energies for Y6 excitons, CT states, and charges. a) Exciton-CT energy offsets and electronic couplings (in $\mathrm{meV}$ ) for $\pi$-stacked molecular pairs extracted from the Y6 crystal structure (Alkyl chains have been removed for clarity). ${ }^{27} \mathbf{b}$ ) Density of states (DOS) for holes (-IE) in a $10 \mathrm{~nm}$ thick model thin film based on molecular dynamics equilibration of the Y6 crystal structure, normalized to unit area. Dashed line indicates the DOS onset determined by photoelectron spectroscopy in air. ${ }^{18}$ c) Average ionisation energy as a function of $\pi$-stacking layer showing differential hole stabilisation for the different lattice positions, which occur in an "...ABBA..." sequence.

The computed electronic couplings and energetics point to a kinetic pathway for exciton breakup (Figure 5, Figure S17). In all cases, the coupling between the lowest exciton and CT states is significant (25-75 meV), with the CT states either equal or lower in energy to the excitonic states (in the molecular geometry of the crystal). These two factors, combined with the low reorganisation energy (estimated as $\sim 0.3 \mathrm{eV}$ based on the reorganisation energies of the individual molecules) imply that exciton break-up is expected, even according to incoherent charge transfer theories. The same electronic coupling parameters/state energies are of course also consistent with the possibility of coherent, or "hot" CT). Indeed, as previously noted, the coupling between excitonic states suggests a high degree of exciton delocalisation, ${ }^{27}$ which is expected to lower the reorganisation barrier for exciton-to-charge conversion.

The coupling of the CT states with the ground state is more variable $(5-80 \mathrm{meV})$, increasing in the order of pair $3<2<1$. This pattern does not simply follow the trend in intermolecular contact area, which is largest for pair 2. The large CT-GS couplings for pairs 1 and 2 are consistent with the rapid recombination observed experimentally. However, the 
apparent independence of $V_{E x-C T}$ and $V_{C T-G S}$ indicates that the recombination can, at least in principle, be overcome by crystal structure engineering to maximise the $V_{E x-C T} / V_{C T-G S}$ ratio.

Our calculations also offer a clue regarding the origin of charge formation in Y6. In pairs 1 and 2, there is a clear energetic preference $(\sim 0.15-0.3 \mathrm{eV})$ for which of the two possible CT states is formed, both of which place the positive and negative charges on the same lattice positions. In pair 3, the molecules occupy equivalent lattice positions, and the difference in CT state energy becomes negligible. It is also striking that pair 3 exhibits an end-on stacking arrangement common to indacenodithienothiophene (IDT) acceptors, ${ }^{27,54,55}$ whereas the remaining two possess extensive contact between the neighbouring chromophores - which is very different to standard FREAs. These observations indicate that although the two crystal positions in Y6 are chemically identical, the packing geometry results in two distinct polarisation environments, creating a donor-acceptor system through supramolecular, rather than synthetic means. This interpretation also explains the significant recombination observed experimentally: the alternation of "donor" and "acceptor" positions on the scale of the unit cell is akin to an extremely intermixed blend system - meaning that both charge formation and recombination are extremely rapid.

The energy levels calculated for free charges also reveal the presence of distinct polarisation environments. Figure 5b shows the ionisation energy (IE) distributions calculated for a Y6 thin film using the long-range embedding procedure available in VOTCA. ${ }^{56-62}$ Electron affinity (EA) is also shown in Fig S13. The input structure for these calculations was taken from a molecular dynamics equilibration of the Y6 crystal structure. ${ }^{27}$ The energetics of molecules occupying different crystal positions reveal an underlying bimodality in the density of states, particularly for holes. The presence of these distinct distributions confirm the presence of a driving force for charge formation inherent to the Y6 packing structure, on the order of the exciton binding energy.

Our calculations reveal equal energy exciton-CT manifolds (as a result from differential polarisation), strong exciton-CT coupling, and a flat energetic landscape for electron transport. Previous theoretical work on Y6 has demonstrated strong electronic coupling for electron and hole transfer in Y6. Taken together, these observations strongly suggest the existence of a kinetic pathway for exciton-to-free charge conversion in Y6. 
We propose a mechanism for how recombination is suppressed in current Y6 blends, drawing on studies invoking energy level bending at the donor/acceptor interface as a result of long-range interactions of charges with molecular quadrupole moments. ${ }^{18,25,56,63-65}$ In an excitonic picture - where charges only form at the interface - these fields assist CT dissociation and suppress non-geminate recombination. However, these benefits come at the expense of exciton dissociation rates, resulting in an intrinsic limit for the ionisation energy offset between the neat materials. ${ }^{18}$ When free charges are intrinsically generated in Y6, not only is the interfacial exciton dissociation barrier bypassed, but the level bending defines an attractive potential for holes, which are pulled towards the interface before leaving the acceptor material, while electrons are repelled. Under this interpretation, champion efficiency systems, such as PM6/Y6, benefit from both exciton- and charge-funnelling effects: excitons in PM6 are funnelled to Y6 via resonant energy transfer, ${ }^{18,66}$ while level bending pulls holes from Y6 into the polymer domain, and suppresses recombination thereafter. We can test whether quadrupolar fields affect charge recombination in Y6 by comparing charge and exciton kinetics of neat Y6, to a film of Y6 blended with $20 \%$ weight ratio PCBM (a ratio present in highly efficient ternary devices ${ }^{5}$ ). The energy levels of PCBM mean that both electron and hole transfer from Y6 to PCBM are prohibited. Any reduction in charge recombination rate will therefore be due to interfacial level bending (one would expect the effect of increased crystallinity in Y6 due to the PCBM would increase recombination, rather than decrease). Figure S16 shows that, as predicted, there is a small reduction in charge recombination rate, and concurrent reduction of exciton population, when PCBM introduced.

\section{Implications for Future OPV Systems}

Efficient intrinsic charge generation, in the FREA small molecule Y6, has broad implications for OPV device design. Considering a population of excitons and free charges in neat Y6 necessitates a more nuanced understanding of the simplified four step process - exciton generation, exciton transport, charge separation at a heterojunction, charge transport - that has previously dominated OPV discussion. Exciton diffusion length is no longer a simple function of how fast a lone exciton travels and how long it lives - interconversion into free charges and the reverse process must also be considered. The ease of charge separation, but concurrent rapid bimolecular charge recombination, necessitate a renewed focus on reducing such recombination. This hints that for current devices the key purpose of the bulk heterojunction is 
more to reduce bimolecular recombination, than to split excitons. Efforts focussed on simultaneously maximising intrinsic PLQE and charge generation should improve OPV device open circuit voltages - which have yet to achieve parity with inorganic photovoltaics. Specific directions of study include: improving crystal packing to further enhance dielectric constants and hence free charge fraction; tuning energy levels through packing structures and/or molecular orientation rather than different compounds; and crystal structure engineering to minimise CT-ground state coupling.

We have shown, through intensity-dependent PLQE measurements, that free charges exist in neat films of the molecular semiconductor Y6, at illumination intensities below 1 sun. We have corroborated this measurement with optically-pumped time-resolved terahertz, ultrafast photoluminescence up-conversion and transient absorption spectroscopy. Our data can be explained by simple kinetic models, along with a new quantum mechanical picture of exciton-CT state coupling and free charge energetics. We have outlined the implications of this finding for our understanding of the photovoltaic process, and the mechanism of action for current champion efficiency devices, and have suggested further avenues of study for enhancing efficiency in future devices. These findings open up the potential to think beyond the bulk heterojunction, and revisit the possibility of fabricating efficient doped organic $\mathrm{p}-\mathrm{n}$ junctions.

\section{METHODS}

General. UV-Vis absorption spectra were obtained using Carry 5000 (Agilent) and a Cary 50 Bio UV-vis spectrometer in the range $190-1100 \mathrm{~nm}$. Photoluminescence spectra were obtained using a Cary Eclipse (Varian). Photoluminescence quantum efficiency measurements were obtained in an integrating sphere using the method of de Mello et al. ${ }^{67} \mathrm{~A}$ Q-switched frequency doubled ND:YAg laser with output wavelength $532 \mathrm{~nm}$, pulse-length $600 \mathrm{ps}$, and rep-rate set to $25 \mathrm{kHz}$ was used to excite the sample for the intensity dependent PLQE measurements. Sample film thicknesses were performed using a Dektak profilometer. Thin films of $\mathrm{Y} 6$, and $\mathrm{Y} 6$ blends were spin-coated onto quartz spectrosil substrates in a glovebox from $11 \mathrm{mg} / \mathrm{ml}$ chloroform solutions, at a spin-speed of $3000 \mathrm{rpm}$. Solid solutions of Y6:polystyrene were prepared in a 1:50 weight ratio, and spincoated in a glovebox at $800 \mathrm{rpm}$.

Device fabrication. Indium tin oxide-patterned glass substrates were cleaned by sequential sonication in acetone and propan-2-ol and subjected to ozone treatment. A $35 \mathrm{~nm}$ film of PEDOT:PSS was spin coated in air from aqueous solution and baked on a hotplate at $110^{\circ} \mathrm{C}$ 
for 15 minutes. A $30 \mathrm{~nm}$ layer of Y6 was spin coated from a chlorobenzene solution in a nitrogen-filled glovebox $\left(0.1 \mathrm{ppm} \mathrm{O}_{2}, 0.0 \mathrm{ppm} \mathrm{H}_{2} \mathrm{O}\right)$. The cathode of $1 \mathrm{~nm} \mathrm{LiF}$ and $90 \mathrm{~nm} \mathrm{Al}$ was deposited by vacuum thermal evaporation at pressure $\sim 10^{-6}$ mbar through a shadow mask to define $6 \mathrm{~mm}^{2}$ pixels. The devices based on PTB7-Th:Y6 were fabricated with a normal structure as ITO glass/PEDOT:PSS/PTB7-Th:Y6/PNDIT-F3N/Ag. Patterned ITO glass was precleaned in an ultrasonic bath with deionized water, acetone and isopropanol, and treated in an ultraviolet-ozone chamber (Jelight Company, USA) for 20 min. PEDOT:PSS was spincoated on the pre-cleaned ITO at $5000 \mathrm{rpm}$, followed by baking at $150{ }^{\circ} \mathrm{C}$ for $15 \mathrm{~min}$. Then, the active layers were spin-coated on PEDOT:PSS (PTB7-Th:Y6, X:12 $\mathrm{mg} \mathrm{mL}^{-1}$ in chloroform, $2500 \mathrm{rpm})$. Afterwards, the PNDIT-F3N solution $\left(0.5 \mathrm{mg} \mathrm{mL}^{-1}\right.$ in methanol) was spin-coated on the active layer at $2000 \mathrm{rpm}$. Finally, Ag electrode (ca. $80 \mathrm{~nm}$ ) was slowly evaporated onto the surface of the underneath layer under vacuum (ca. $10^{-5} \mathrm{~Pa}$ ). The devices were not masked and the active area of devices were $4 \mathrm{~mm}^{2}$.

Device characterisation. Photovoltaic device JV characteristics were measured using a solar simulator and source-measure unit. Light intensity was determined using a calibrated silicon diode. Light intensity was varied by adjusting the distance between source and device under test and also by use of neutral density filters. EQE spectra of PTB7-Th:Y6 devices were obtained using a Solar Cell Spectral Response Measurement System QE-R3011 (Enlitech Co.).

Transient absorption spectroscopy measurements were performed using a homebuilt experimental setup illuminated by an amplified Ti:sapphire laser, with pulse durations of 100$150 \mathrm{fs}$, centred around $800 \mathrm{~nm}$ and at a repetition rate of $3 \mathrm{kHz}$. The excitation pulses are generated either using this fundamental, or by using an optical parametric amplifier (TOPAS) with the $800 \mathrm{~nm}$ fundamental input and then chopped at $f / 2(1.5 \mathrm{kHz})$. Photoexcitations in the materials were probed via a broadband white light continuum generated by focusing a portion of the fundamental to an undoped YAG (Yttrium Aluminium Garnet) crystal. Pump-probe polarizations were kept under magic angle $\left(54.7^{\circ}\right)$ configuration to avoid orientational dynamics. After passing through the photoexcited sample, the probe pulses were spectrally dispersed using a prism spectrometer and are then collected using a CMOS camera (visible components) or an InGaAs photodiode array (IR components). The time resolution is obtained via introducing time delays in the pump path which is achieved using a retroreflector connected to a motorized translational stage. The differential transmission signals at various time delays are calculated from the sequential probe shots corresponding to the pump on versus off. For typical measurements, 8000 shots were averaged at each time point and were repeated at least four times. To avoid degradation, all the samples were measured under a vacuum, or inert nitrogen environment.

Time-resolved terahertz spectroscopy was performed using a dual lock-in technique, similar to that described by Tiwana et al. ${ }^{68} \mathrm{THz}$ pulses are generated by an amplified Ti:sapphire laser, with pulse durations of 100-150 fs, centred around $800 \mathrm{~nm}$, generated at a repetition rate of 3 $\mathrm{kHz}$ chopped to $1.5 \mathrm{kHz}$, incident onto a $1 \mathrm{~mm}$ thick ZnTe crystal. THz pulses are measured by balanced photodetectors using electrooptic sampling in a separate ZnTe crystal. The $800 \mathrm{~nm}$ 'optical' pump is chopped at $750 \mathrm{~Hz}$, and incident on the sample with a spot-size diameter of 4-5 $\mathrm{mm}$. The THz probe diameter was measured as $\sim 1-2 \mathrm{~mm}$. The entire THz beam path is enclosed in a cavity pumped with dry air, with humidity monitored to ensure $\%$ water remains 
below $2 \%$. The sample is enclosed in a nitrogen environment to avoid laser degradation and is excited from the glass substrate side. $\mathrm{THz}$ conductivity was calculated ${ }^{68}$ as:

$$
\Delta \sigma=-\frac{\varepsilon_{0} c\left(n_{A}+n_{B}\right)}{L} \frac{\Delta T}{T}
$$

Where $\varepsilon_{0}$ is the vacuum permittivity, $c$ is the speed of light, $n_{A}$ and $n_{B}$ are the refractive indices of the media on both sides of the sample in the THz region - quartz, and air, $L$ is the sample thickness, and $\frac{\Delta T}{T}$ the measured change in $\mathrm{THz}$ transmittance.

Ultrafast transient photoluminescence dynamics were measured using a photoluminescence up-conversion technique. The setup's light source is a Ytterbium fiber laser (Tangerine SP, Amplitude Systemes) operating at $44 \mathrm{kHz}$ and generating $150 \mathrm{fs}$ pulses. The laser output was split into pump and gate parts. The pump was frequency-doubled to $515 \mathrm{~nm}$ using a BBO crystal and focused to a $50 \mu \mathrm{m}^{2}$ spot to excite the sample. The sample fluorescence was collimated and refocused on a nonlinear mixing crystal $\left(1 \mathrm{~mm} \mathrm{BBO}, \theta=32^{\circ}\right)$ by a pair of aluminium-coated off-axis parabolic mirrors. The crystal angle is set for the phase-matching condition (type I) to produce sum frequency signals at $480 \mathrm{~nm}$ from mixing the $1030 \mathrm{~nm}$ gate and $900 \mathrm{~nm}$ photoluminescence. Two achromatic lenses collimated and focussed the upconverted signals onto the spectrometer (Princeton Instruments SP 2150). Signals were detected by an intensified CCD camera (Princeton Instruments, PIMAX3). A combination of $550 \mathrm{~nm}$ long- and $800 \mathrm{~nm}$ short-pass filters were used to remove residual excitation and 1030 $\mathrm{nm}$ gate light, respectively. The time delay between pump and gate beams was controlled via a motorized optical delay line on the excitation beam path. For each delay time, 240000 shots were accumulated. Samples were measured under a vacuum environment.

Density functional theory calculations were performed using Gaussian $09^{69}$ with an ultrafine integration grid, and the accuracy of two-electron integrals increased to $10^{11}$. Optimized geometries were confirmed by calculation of the associated vibrational modes, which revealed no imaginary frequencies in all cases.

Electronic Coupling calculations were conducted on pairs of molecules extracted from the X-ray crystal structure, with alkyl chains truncated to ethyl. These calculations used the long-range corrected CAM-B3LYP ${ }^{70}$ exchange-correlation functional in combination with the $6-31+G(d, p)$ basis set. Linear response TD-DFT calculations for excited states were performed within the Tamm-Dankoff approximation (TDA). Dielectric stabilisation was treated using a polarisable continuum model with $\epsilon=5.0$, based on the experimentally-determined value. ${ }^{29,71-}$ 74 To treat CT states, the exchange-correlation functional was range-tuned according to the established non-empirical procedure ${ }^{71-74}$ resulting in an optimal range-separation parameter of $0.0120 \mathrm{Bohr}^{-1}$. Electronic couplings and exciton/CT energetics were accessed from the DFT output using our previously described projective coupling method. ${ }^{52,53}$

Density of States calculations for electrons and holes were performed using the longrange embedding procedure available in VOTCA-CTP. ${ }^{56-62}$ Molecular charge densities and polarizabilities for the ground state, cation, and anion were calculated using B3LYP/6$311 \mathrm{G}(\mathrm{d}, \mathrm{p})$. The charge densities were used to fit distributed atomic multipoles (specifically, atomic charges, dipoles, and quadrupoles) using GDMA (version 2.3 ${ }^{75}$. Polarizabilities for atoms in alkyl chains were taken directly from the set of Thole polarizabilities. ${ }^{76}$ Atomic polarizabilities for atoms in the Y6 chromophore were obtained by scaling Thole 
polarizabilities to match the DFT-calculated polarisable volume of the core. ${ }^{56}$ Solid state corrections to the gas-phase IE/EA were calculated using the long-range Ewald summation scheme in VOTCA-CTP. These calculations employed a molecular dynamics equilibrated $8 \times$ $8 \times 6$ supercell constructed from the Y6 crystal structure, ${ }^{27}$ with the $\pi-\pi$ stacking direction aligned with the $z$-axis, to match the experimentally observed face-on packing preference relative to the substrate. This simulation cell was used to mimic a $\sim 10 \mathrm{~nm}$ thin film by including a $\sim 20 \mathrm{~nm}$ vacuum buffer in the $z$ direction and applying the shape term for a periodically repeated supercell in the xy plane. The interaction cut-offs for electrostatic and polarization interactions were 8 and $6 \mathrm{~nm}$, respectively.

Molecular dynamics simulations were conducted using the Y6 crystal structure with the missing atoms of the alkyl side chains built in. The system containing $8 \times 8 \times 6$ unit cells was generated using Maestro ${ }^{77}$ in the Schrödinger Material Science Suite, and then prepared for molecular dynamics simulation with the OPLS3e force field using the System Builder in Desmond. $^{78,79}$ The prepared system first underwent 100 ps of Brownian minimization, followed by 1 ns of equilibration using NPT molecular dynamics simulation at $1 \mathrm{fs}$ timestep, $300 \mathrm{~K}$ and 1.01325 bar. A further $10 \mathrm{ps}$ molecular dynamics simulation was conducted to collect 200 frames of trajectories at 50 fs time interval for analysis.

\section{Acknowledgements}

M.B.P., P.A.H., A.I, I.W., R.R.T., K.E.T., N.J..L.K.D, K.C and J.M.H. acknowledge support from the MacDiarmid Institute of New Zealand. P.A.H., A.I, I.W., R.R.T., K.E.T., N.J..L.K.D, K.C and J.M.H. acknowledge funding from the Marsden Fund of NZ. M.B.P and N.J.L.K.D. acknowledge support from the Royal Society of New Zealand. P.A.H. would like to acknowledge the support of Professor Denis Andrienko, Dr Anastasia Markina and Dr. Carl Poelking for support in utilising VOTCA software.

\section{References}

1. Riede, M., Spoltore, D. \& Leo, K. Organic Solar Cells - The Path to Commercial Success. Adv. Energy Mater. 11, 1-10 (2021).

2. Burlingame, Q. et al. Intrinsically stable organic solar cells under high-intensity illumination. Nature 573, (2019).

3. Hou, J., Inganas, O., Friend, R. H. \& Gao, F. Organic solar cells based on nonfullerene acceptors. Nature Materials 119-128 (2018) doi:10.1038/NMAT5063.

4. Halls, J. J. M. et al. Efficient photodiodes from interpenetrating polymer networks. Nature vol. 376 498-500 (1995).

5. Wen, Z. C., Yin, H. \& Hao, X. T. Recent progress of PM6:Y6-based high efficiency organic solar cells. Surfaces and Interfaces 23, 100921 (2021).

6. Cui, Y. et al. Single-Junction Organic Photovoltaic Cells with Approaching 18\% 
571

7. Lin, Y. et al. $17.1 \%$ Efficient Single-Junction Organic Solar Cells Enabled by n-Type Doping of the Bulk-Heterojunction. Adv. Sci. 1903419, 1-9 (2020).

8. Wang, Y. et al. High-Efficiency Perovskite Quantum Dot Hybrid Nonfullerene Organic Solar Cells with Near-Zero Driving Force. Adv. Mater. 32, (2020).

9. Kerremans, R. et al. The Optical Constants of Solution-Processed SemiconductorsNew Challenges with Perovskites and Non-Fullerene Acceptors. Adv. Opt. Mater. 8, (2020).

10. Few, S., Frost, J. M. \& Nelson, J. Models of charge pair generation in organic solar cells. Phys. Chem. Chem. Phys. 17, 2311-2325 (2015).

11. Chen, S., Tsang, S. W., Lai, T. H., Reynolds, J. R. \& So, F. Dielectric effect on the photovoltage loss in organic photovoltaic cells. Adv. Mater. 26, 6125-6131 (2014).

12. Tang, C. W. Two-layer organic photovoltaic cell. Appl. Phys. Lett. 48, (1986).

13. Kozlov, O. V. et al. Ultrafast Exciton-to-Polaron Conversion in Densely Packed Small Organic Semiconducting Molecules. Adv. Opt. Mater. 5, 1-7 (2017).

14. Chandran, H. T. et al. Direct Free Carrier Photogeneration in Single Layer and Stacked Organic Photovoltaic Devices. Adv. Mater. 29, (2017).

15. Nikolis, V. C. et al. Field Effect versus Driving Force: Charge Generation in SmallMolecule Organic Solar Cells. Adv. Energy Mater. 10, (2020).

16. Wang, R. et al. Charge Separation from an Intra-Moiety Intermediate State in the High-Performance PM6:Y6 Organic Photovoltaic Blend. J. Am. Chem. Soc. 142, 12751-12759 (2020).

17. Benduhn, J. et al. Intrinsic non-radiative voltage losses in fullerene-based organic solar cells. Nat. Energy 2, (2017).

18. Karuthedath, S. et al. Intrinsic efficiency limits in low-bandgap non-fullerene acceptor organic solar cells. Nat. Mater. (2020) doi:10.1038/s41563-020-00835-x.

19. Xiao, F., Li, C., Sun, J. \& Zhang, L. Knowledge domain and emerging trends in organic photovoltaic technology: A scientometric review based on citespace analysis. Front. Chem. 5, 1-12 (2017). 
20. Yuan, J. et al. Single-Junction Organic Solar Cell with over 15\% Efficiency Using Fused-Ring Acceptor with Electron-Deficient Core. Joule (2019) doi:10.1016/j.joule.2019.01.004.

21. Lin, Y. et al. An electron acceptor challenging fullerenes for efficient polymer solar cells. Adv. Mater. (2015) doi:10.1002/adma.201404317.

22. Yan, C. et al. Non-fullerene acceptors for organic solar cells. Nat. Rev. Mater. 3, (2018).

23. Wang, J. \& Zhan, X. Fused-Ring Electron Acceptors for Photovoltaics and beyond. Acc. Chem. Res. 54, (2021).

24. Cheng, P., Li, G., Zhan, X. \& Yang, Y. Next-generation organic photovoltaics based on non-fullerene acceptors. Nat. Photonics 12, (2018).

25. Perdigón-Toro, L. et al. Barrierless Free Charge Generation in the High-Performance PM6:Y6 Bulk Heterojunction Non-Fullerene Solar Cell. Adv. Mater. 32, (2020).

26. Zhu, X. et al. Self-Stimulated Dissociation in Non-Fullerene Organic BulkHeterojunction Solar Cells Self-Stimulated Dissociation in Non-Fullerene Organic Bulk-Heterojunction Solar Cells. Joule 4, 1-15 (2020).

27. Zhang, G. et al. Delocalization of exciton and electron wavefunction in non-fullerene acceptor molecules enables efficient organic solar cells. Nat. Commun. 11, 1-10 (2020).

28. Liu, J. et al. Charge separation boosts exciton diffusion in fused ring electron acceptors. J. Mater. Chem. A 8, (2020).

29. Kerremans, R. et al. The Optical Constants of Solution-Processed SemiconductorsNew Challenges with Perovskites and Non-Fullerene Acceptors. Adv. Opt. Mater. 2000319, (2020).

30. Park, S. Y. et al. Photophysical pathways in efficient bilayer organic solar cells: The importance of interlayer energy transfer. Nano Energy 84, (2021).

31. Lin, Q. et al. Electro-optics of perovskite solar cells. Nat. Photonics 9, 106-112 (2014).

32. Li, H. et al. On the Study of Exciton Binding Energy with Direct Charge Generation in 
629

630

631

632

633

634

635

636

637

638

639

640

641

642

643

644

645

646

647

648

649

650

651

652

653

654

655

656

33. Mondelli, P. et al. Meta-analysis: The molecular organization of non-fullerene acceptors. Mater. Horizons 7, 1062-1072 (2020).

34. Northrup, J. E. Mobility enhancement in polymer organic semiconductors arising from increased interconnectivity at the level of polymer segments. Appl. Phys. Lett. 106, (2015).

35. Gélinas, S. et al. Ultrafast Long-Range Charge Separation in Organic Semiconductor Photovoltaic Diodes. Science (80-. ). 343, 512-517 (2014).

36. Westenhoff, S. et al. Charge recombination in organic photovoltaic devices with high open-circuit voltages. J. Am. Chem. Soc. 130, 13653-13658 (2008).

37. Bakulin, A. A. et al. The role of driving energy and delocalized states for charge separation in organic semiconductors. Science (80-. ). 335, (2012).

38. Gélinas, S. et al. Ultrafast long-range charge separation in organic semiconductor photovoltaic diodes. Science (80-. ). 343, 512-516 (2014).

39. Gillett, A. J. et al. The role of charge recombination to spin-triplet excitons in nonfullerene acceptor organic solar cells. ArXiv (2020).

40. Krauspe, P. et al. Terahertz short-range mobilities in neat and intermixed regions of polymer:fullerene blends with controlled phase morphology. J. Mater. Chem. A 6 , 22301-22309 (2018).

41. Hendry, E., Schins, J. M., Candeias, L. P., Siebbeles, L. D. A. \& Bonn, M. Efficiency of exciton and charge carrier photogeneration in a semiconducting polymer. Phys. Rev. Lett. 92, 1-4 (2004).

42. Cooke, D. G., Krebs, F. C. \& Jepsen, P. U. Multi-THz spectroscopy of mobile charge carriers in P3HT:PCBM on a sub-100 fs time scale. Phys. Chem. Interfaces Nanomater. XII 8811, 88111H (2013).

43. Sun, Y. et al. Electronic processes investigation from ultrafast terahertz in photovoltaic DPPDTT-PCBM films. Sol. Energy Mater. Sol. Cells 215, 110684 (2020).

44. Richter, J. M. et al. Enhancing photoluminescence yields in lead halide perovskites by photon recycling and light out-coupling. Nat. Commun. (2016) 
doi:10.1038/ncomms13941.

45. Hou, Y. et al. Charge separation, charge recombination, long-lived charge transfer state formation and intersystem crossing in organic electron donor/acceptor dyads. $J$. Mater. Chem. C 7, 12048-12074 (2019).

46. Yablonovitch, E., Miller, O. D. \& Kurtz, S. R. A great solar cell also needs to be a great LED: External fluorescence leads to new efficiency record. in AIP Conference Proceedings vol. 1519 (2013).

47. Kyaw, A. K. K. et al. Intensity dependence of current-voltage characteristics and recombination in high-efficiency solution-processed small-molecule solar cells. ACS Nano 7, (2013).

48. Koster, L. J. A., Kemerink, M., Wienk, M. M., Maturová, K. \& Janssen, R. A. J. Quantifying bimolecular recombination losses in organic bulk heterojunction solar cells. Adv. Mater. 23, (2011).

49. Koster, L. J. A., Mihailetchi, V. D., Xie, H. \& Blom, P. W. M. Origin of the light intensity dependence of the short-circuit current of polymer/fullerene solar cells. Appl. Phys. Lett. 87, (2005).

50. Mihailetchi, V. D., Wildeman, J. \& Blom, P. W. M. Space-charge limited photocurrent. Phys. Rev. Lett. 94, (2005).

51. Liao, S. H., Jhuo, H. J., Cheng, Y. S. \& Chen, S. A. Fullerene derivative-doped zinc oxide nanofilm as the cathode of inverted polymer solar cells with low-bandgap polymer (PTB7-Th) for high performance. Adv. Mater. 25, (2013).

52. Hume, P. A. \& Hodgkiss, J. M. A Projective Method for the Calculation of ExcitedState Electronic Coupling: Isolating Charge Transfer/Recombination Processes in Organic Photovoltaics. J. Phys. Chem. A 124, 591-600 (2020).

53. Hume, P. A., Jiao, W. \& Hodgkiss, J. M. Long-range exciton diffusion in a nonfullerene acceptor: approaching the incoherent limit. J. Mater. Chem. C 1419-1428 (2021) doi:10.1039/d0tc05697a.

54. Aldrich, T. J. et al. Fluorination Effects on Indacenodithienothiophene Acceptor Packing and Electronic Structure, End-Group Redistribution, and Solar Cell Photovoltaic Response. J. Am. Chem. Soc. (2019) doi:10.1021/jacs.8b13653. 
55. Lai, H. et al. Isomer-free: Precise Positioning of Chlorine-Induced Interpenetrating Charge Transfer for Elevated Solar Conversion. iScience 17, 302-314 (2019).

56. Poelking, C. \& Andrienko, D. Long-Range Embedding of Molecular Ions and Excitations in a Polarizable Molecular Environment. J. Chem. Theory Comput. 12, 4516-4523 (2016).

57. Kirkpatrick, J. An approximate method for calculating transfer integrals based on the ZINDO Hamiltonian. Int. J. Quantum Chem. 108, (2008).

58. Rühle, V., Junghans, C., Lukyanov, A., Kremer, K. \& Andrienko, D. Versatile objectoriented toolkit for coarse-graining applications. J. Chem. Theory Comput. 5, (2009).

59. Baumeier, B., Kirkpatrick, J. \& Andrienko, D. Density-functional based determination of intermolecular charge transfer properties for large-scale morphologies. Phys. Chem. Chem. Phys. 12, (2010).

60. Lukyanov, A. \& Andrienko, D. Extracting nondispersive charge carrier mobilities of organic semiconductors from simulations of small systems. Phys. Rev. B - Condens. Matter Mater. Phys. 82, (2010).

61. Rühle, V. et al. Microscopic simulations of charge transport in disordered organic semiconductors. Journal of Chemical Theory and Computation vol. 7 (2011).

62. Kordt, P. \& Andrienko, D. Modeling of Spatially Correlated Energetic Disorder in Organic Semiconductors. J. Chem. Theory Comput. 12, (2016).

63. Poelking, C. \& Andrienko, D. Design rules for organic donor-acceptor heterojunctions: Pathway for charge splitting and detrapping. J. Am. Chem. Soc. 137, 6320-6326 (2015).

64. Schwarze, M. et al. Impact of molecular quadrupole moments on the energy levels at organic heterojunctions. Nat. Commun. 10, 1-9 (2019).

65. Poelking, C. et al. Impact of mesoscale order on open-circuit voltage in organic solar cells. Nat. Mater. 14, 434-439 (2015).

66. Park, S. Y., Chandrabose, S. \& Price, M. B. Photophysical pathways in efficient bilayer organic solar cells: the importance of interlayer energy transfer. Nano Energy (2021). 
67. de Mello, J. C., Wittmann, H. F. \& Friend, R. H. An improved experimental determination of external photoluminescence quantum efficiency. Adv. Mater. 9, 230232 (1997).

68. Tiwana, P., Parkinson, P., Johnston, M. B., Snaith, H. J. \& Herz, L. M. Ultrafast terahertz conductivity dynamics in mesoporous $\mathrm{TiO} 2$ : Influence of dye sensitization and surface treatment in solid-state dye-sensitized solar cells. J. Phys. Chem. C 114, 1365-1371 (2010).

69. Frisch, M. J. et al. Gaussian09 Revision D.01, Gaussian Inc. Wallingford CT. Gaussian 09 Revision C.01 (2010).

70. Yanai, T., Tew, D. P. \& Handy, N. C. A new hybrid exchange-correlation functional using the Coulomb-attenuating method (CAM-B3LYP). Chem. Phys. Lett. 393, (2004).

71. Kronik, L., Stein, T., Refaely-Abramson, S. \& Baer, R. Excitation gaps of finite-sized systems from optimally tuned range-separated hybrid functionals. Journal of Chemical Theory and Computation vol. 8 (2012).

72. Sun, H. et al. Ionization Energies, Electron Affinities, and Polarization Energies of Organic Molecular Crystals: Quantitative Estimations from a Polarizable Continuum Model (PCM)-Tuned Range-Separated Density Functional Approach. J. Chem. Theory Comput. 12, (2016).

73. Refaely-Abramson, S. et al. Gap renormalization of molecular crystals from densityfunctional theory. Phys. Rev. B - Condens. Matter Mater. Phys. 88, (2013).

74. Chen, X. K. \& Brédas, J. L. Voltage Losses in Organic Solar Cells: Understanding the Contributions of Intramolecular Vibrations to Nonradiative Recombinations. Adv. Energy Mater. 8, (2018).

75. Stone, A. J. Distributed multipole analysis: Stability for large basis sets. J. Chem. Theory Comput. 1, (2005).

76. Van Duijnen, P. T. \& Swart, M. Molecular and atomic polarizabilities: Thole's model revisited. J. Phys. Chem. A 102, (1998).

77. Schrödinger Release 2020-2: Maestro. (2020). 
79. Schrödinger Release 2020-2: Desmond Molecular Dynamics System. (2020). Proceedings of the Conference on High Performance Computing Networking, Storage and Analysis, SC '09 (2009). doi:10.1145/1654059.1654099. 
Figures
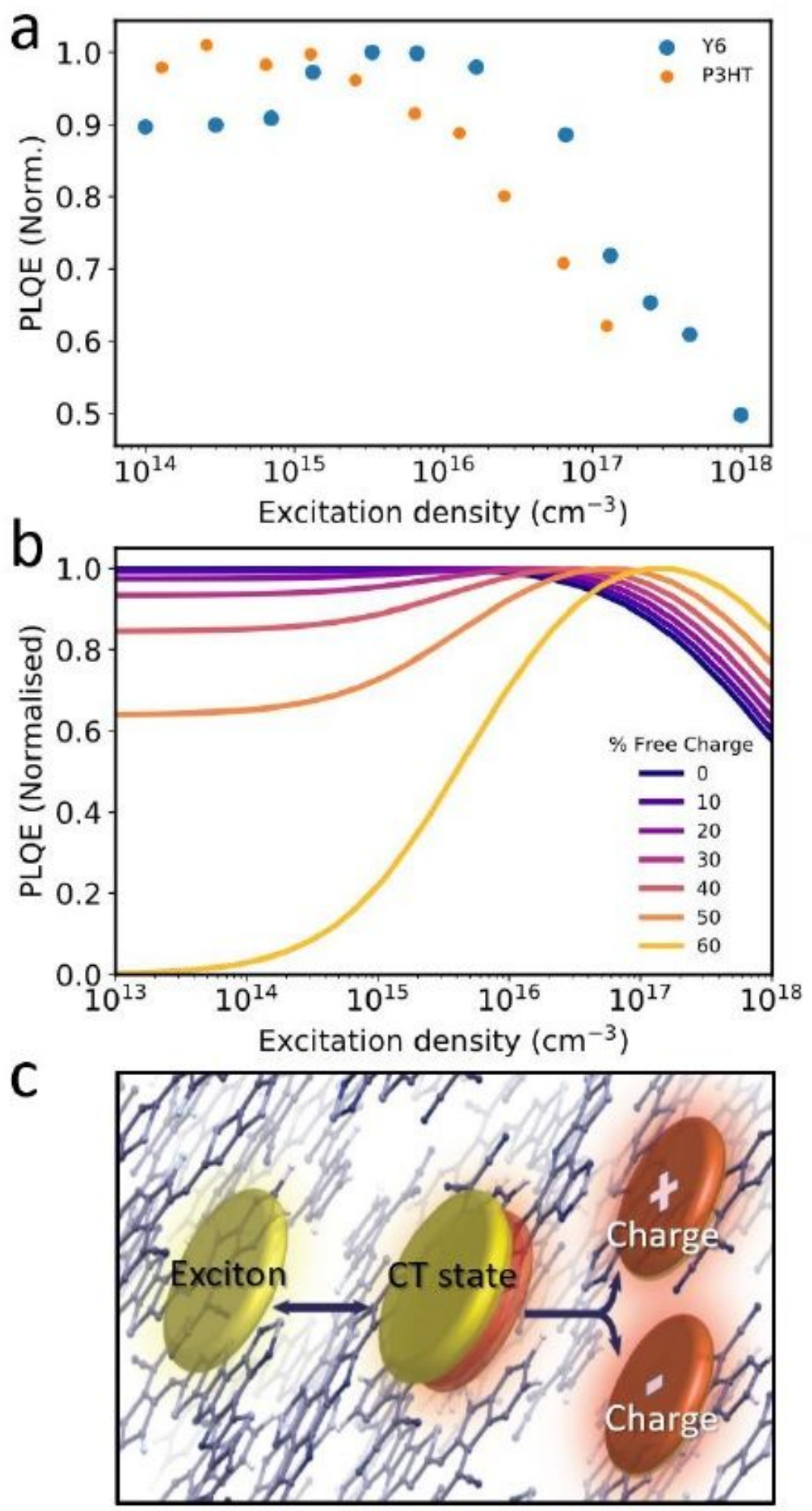

\section{Figure 1}

Intensity-dependent photoluminescence quantum efficiency of Y6. a) Experimentally determined PLQE of a neat film of $\mathrm{Y} 6$ on glass substrate in an integrating sphere (normalised). The sample was excited by 600 ps pulses of $532 \mathrm{~nm}$ light. The PLQE peaks at 1\% at 1016 excitations/cm3. Figure S1 gives an 
indication of the error associated with the data. Intensity dependent PLQE of a film of P3HT is shown as orange circles, as comparison, to illustrate the expected response. b) Simulation of intensity-dependent PLQE values showing effect of increasing (orange) or decreasing (blue) radiative bimolecular recombination (from prompt free charge fraction, calculated using Model 2 detailed in the SI). c) Graphic representation of exciton-CT state-free charge equilibrium in Y6.
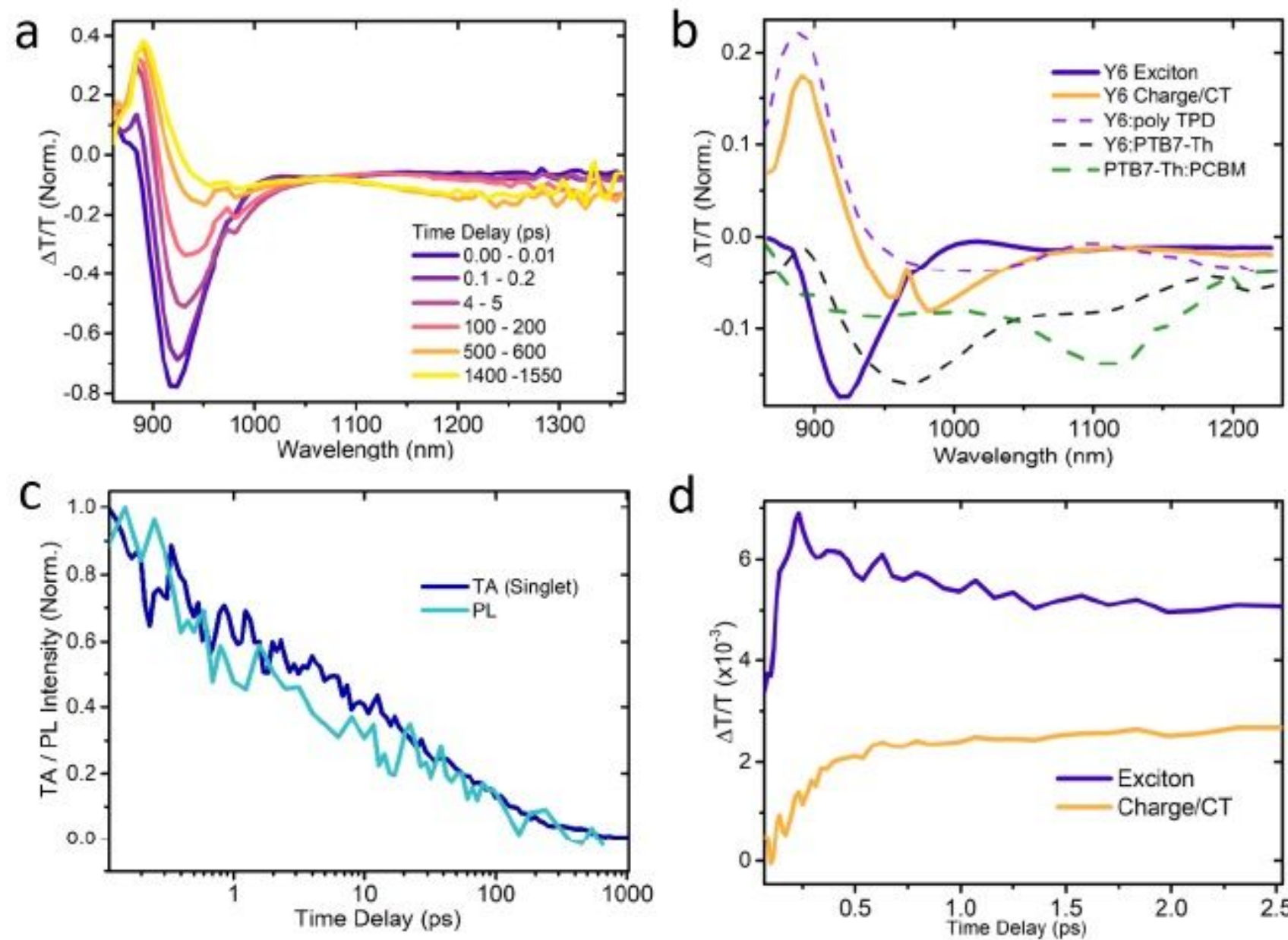

d
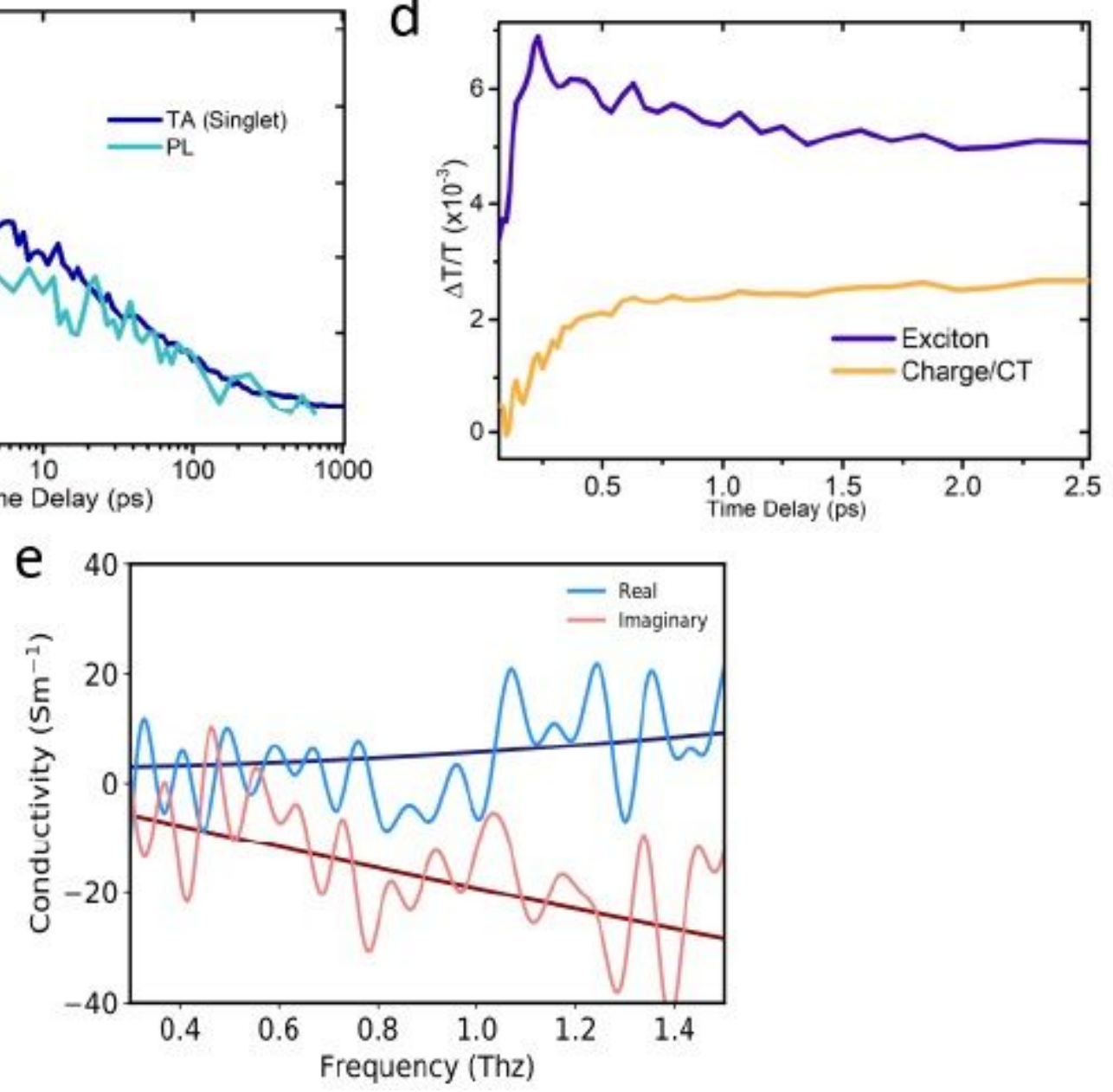

Figure 2 
Ultrafast transient absorption spectra and kinetics of singlets and free charges in neat $Y 6$, and optical pump terahertz probe conductivity. a) Transient absorption spectra, normalised to the absolute area integral, of a neat film of $\mathrm{Y} 6$ at different time delays. $700 \mathrm{~nm}, 200 \mathrm{fs}$ pump pulse, excitation density = 1015 excitations $/ \mathrm{cm} 3$. The spectrum evolves from the exciton, blue line, with a negative peak at $915 \mathrm{~nm}$, to the red-shifted charge/CT state spectrum, orange line. b) Confirmation of charge and exciton transient absorption spectra by comparison of neat species (extracted using genetic algorithm) with species resolved from blends of Y6 with hole and electron accepting materials (PTB7-Th, polyTPD). The green dashed line shows the hole spectra of PTB7-Th blended with PCBM. c) Singlet component from GA of transient absorption (dark blue line), compared with up-converted PL signal (cyan), at an excitation density of $8 \times 1014 \mathrm{~cm}-3 . \mathrm{d}$ ) Initial kinetics of the exciton (blue) versus charge/CT (orange) TA kinetics in the first 2.5 ps after photoexcitation. The charge species shows a rise concomitant with the fast initial decay of the singlet species. e) Excited-state terahertz complex conductivity spectrum, excited with 150 fs, $800 \mathrm{~nm}$ light, giving approximate excitation density 5×1015 excitations/cm3, with a THz probe delay of $\sim 3$ ps. The positive real (light blue) line is indicative that there are charge-like species present in addition to purely excitonic photoexcitations. The experimental data is fit to a modified Drude-Smith model (darker blue and darker red curves). 

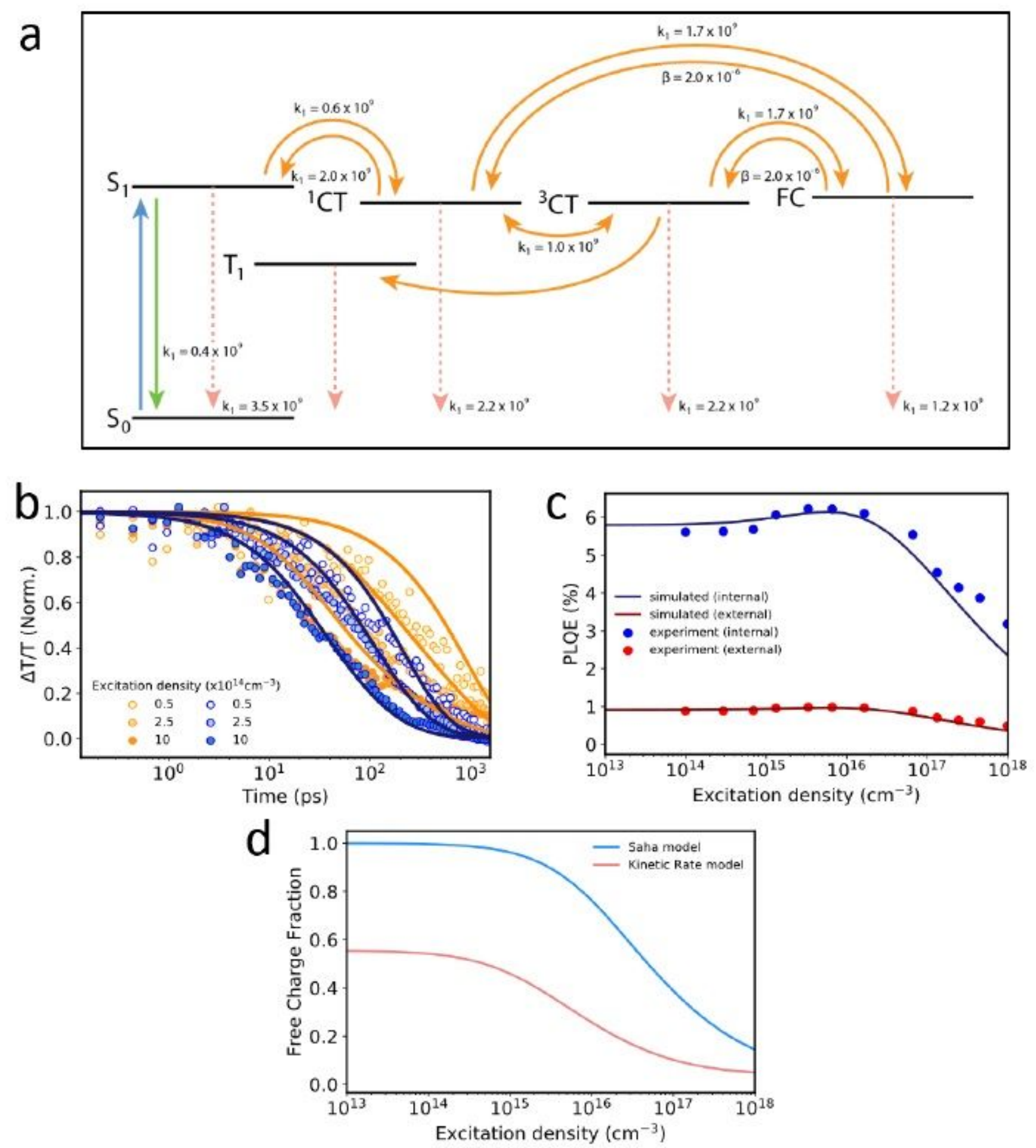

\section{Figure 3}

Kinetic model of charge generation in Y6 with transient absorption, PLQE and predicted steady-state free charge fractions. a) Jablonski diagram highlighting key excited-state pathways, with associated rate constants. Monomolecular, k1, values are given in units s-1, and bimolecular, $\beta$, values given in $\mathrm{cm} 3 \mathrm{~s}-1$. b) Normalised transient absorption kinetics of excitons (blue circles) and charges+CT states (orange circles) at different excitation densities, fitted with a global fit to the kinetic model described in the text and SI 
(solid blue and orange lines). c) Internal (blue) and external (red) PLQE values of Y6 as a function of excitation density, with corresponding simulated values from fits to the transient absorption and intensity dependent PLQE. Internal PLQE is calculated from external PLQE as per Richter et al.44 d) Steady-state free charge fraction as a function of total excitation density calculated from the rate constants gathered from the kinetic model (red line), and from an estimate from the Saha equation (blue line).
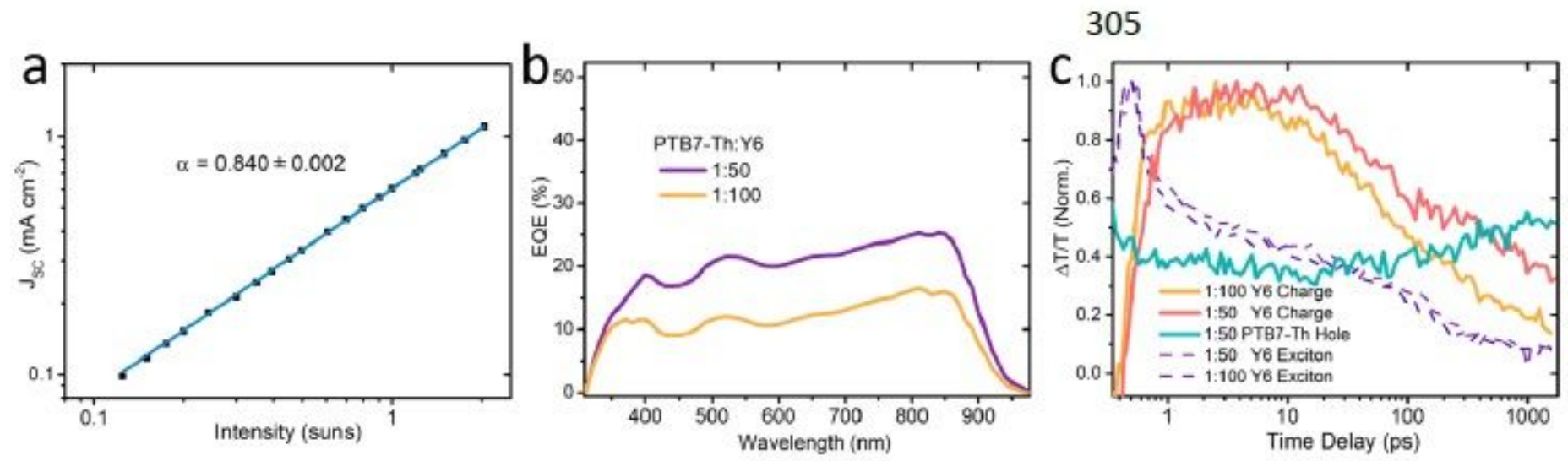

\section{Figure 4}

Single component short circuit current, and low donor active layer device EQEs, and corresponding exciton and charge kinetics. a) Intensity dependent JSC of a monolayer Y6 device. The power exponent fitted to the data between 0.2 and 2 suns is 0.7 , indicating a high number of bimolecular recombination events. b) External quantum efficiencies (EQEs) of devices with active layers consisting of small ratios of donor, PTB7-Th, to acceptor, Y6. For such low donor content, EQEs are unexpectedly high. c) Normalised transient absorption kinetics of exciton and charge species in blends of PTB7-Th:Y6 with ratios of 1:50 and 1:100. Pumped with $800 \mathrm{~nm}, 150 \mathrm{fs}$ pulses at excitation density $1 \times 1014$ excitations/cm3. The exciton kinetics of both films, dashed lines, are matching, as are the rise times of the charge kinetics (orange and red lines), but the 1:50 blend shows slower charge recombination after $20 \mathrm{ps}$. The green-blue line shows the hole kinetics in the 1:50 blend, showing a gradual increase in hole population as excitons and charges in Y6 diffuse to the PTB7-Th. 
a

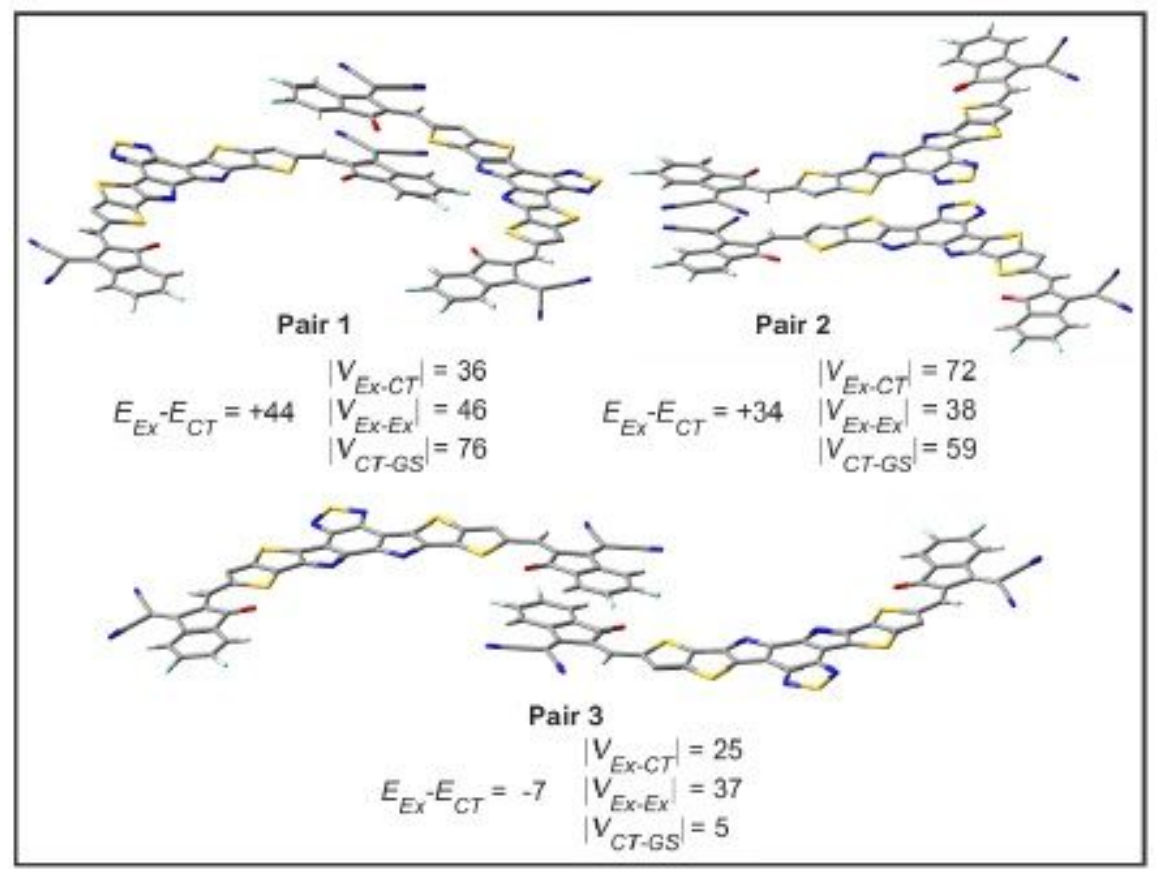

b

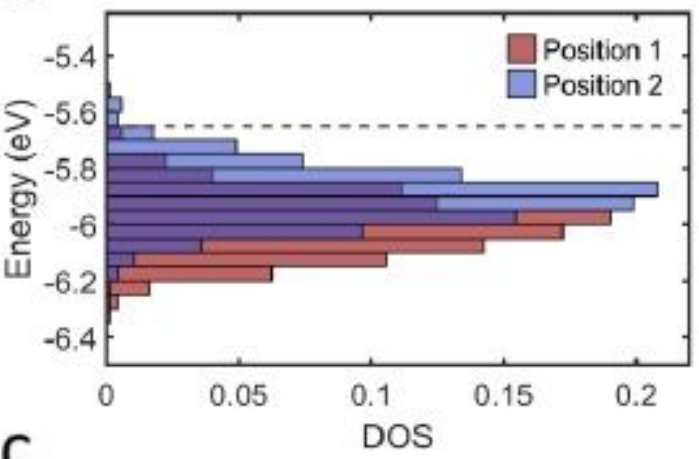

C

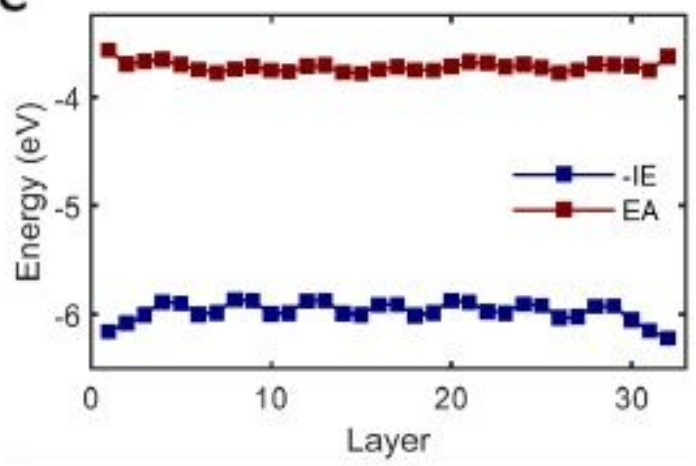

\section{Figure 5}

Electronic couplings and energies for $\mathrm{Y} 6$ excitons, CT states, and charges. a) Exciton-CT energy offsets and electronic couplings (in meV) for $\pi$-stacked molecular pairs extracted from the $\mathrm{Y} 6$ crystal structure (Alkyl chains have been removed for clarity).27 b) Density of states (DOS) for holes (-IE) in a $10 \mathrm{~nm}$ thick model thin film based on molecular dynamics equilibration of the $Y 6$ crystal structure, normalized to unit area. Dashed line indicates the DOS onset determined by photoelectron spectroscopy in air.18 c) Average ionisation energy as a function of $\pi$-stacking layer showing differential hole stabilisation for the different lattice positions, which occur in an "...ABBA..." sequence.

\section{Supplementary Files}

This is a list of supplementary files associated with this preprint. Click to download.

- SupplementaryinformationFreechargegenerationinY6300321.pdf 